\title{
Optimal constraint projection for hyperbolic evolution systems
}

\author{
Michael Holst, ${ }^{1,2}$ Lee Lindblom, ${ }^{1}$ Robert Owen, ${ }^{1}$ Harald P. Pfeiffer, ${ }^{1}$ Mark A. Scheel, ${ }^{1}$ and Lawrence E. Kidder ${ }^{3}$ \\ ${ }^{1}$ Theoretical Astrophysics 130-33, California Institute of Technology, Pasadena, California 91125, USA \\ ${ }^{2}$ Department of Mathematics, University of California at San Diego, USA, La Jolla, California 92093, USA \\ ${ }^{3}$ Center for Radiophysics and Space Research, Cornell University, Ithaca, New York, 14853, USA
}

(Received 2 July 2004; published 13 October 2004)

\begin{abstract}
Techniques are developed for projecting the solutions of symmetric-hyperbolic evolution systems onto the constraint submanifold (the constraint-satisfying subset of the dynamical field space). These optimal projections map a field configuration to the nearest configuration in the constraint submanifold, where distances between configurations are measured with the natural metric on the space of dynamical fields. The construction and use of these projections are illustrated for a new representation of the scalar field equation that exhibits both bulk and boundary generated constraint violations. Numerical simulations on a black hole background show that bulk constraint violations cannot be controlled by constraint-preserving boundary conditions alone, but are effectively controlled by constraint projection. Simulations also show that constraint violations entering through boundaries cannot be controlled by constraint projection alone, but are controlled by constraint-preserving boundary conditions. Numerical solutions to the pathological scalar field system are shown to converge to solutions of a standard representation of the scalar field equation when constraint projection and constraint-preserving boundary conditions are used together.
\end{abstract}

DOI: 10.1103/PhysRevD.70.084017

PACS numbers: 04.25.Dm, 02.60.Cb, 04.20.Cv

\section{INTRODUCTION}

The exponential growth of constraint violations in the evolutions of black hole spacetimes is probably the most critical problem facing the numerical relativity community today. The evolution equations of any self-consistent evolution system with constraints (including Einstein's) ensure that if the constraints are satisfied identically on an initial spacelike surface, they will remain satisfied within the domain of dependence of that surface. This does not mean that small initial violations of the constraints will remain small, or that constraint violations will not flow into the computational domain through timelike boundaries. On the contrary, experience has shown that constraint violations seeded by roundoff or truncation level errors in the initial data tend to grow exponentially in the numerical evolutions of black hole spacetimes (see, e.g., [1-3]). At present these constraintviolating instabilities are the limiting factor preventing these numerical simulations from running for the desired length of time. Finding ways to control the growth of these constraints is therefore our most urgent priority.

Recent work has demonstrated numerically that constraint violations that flow into the computational domain through timelike boundaries can be controlled effectively by the use of special constraint-preserving boundary conditions [4-8]. A number of groups have constructed such boundary conditions for various representations of the Einstein evolution system [5-7,9-16]. However, constraint violations in many evolution systems (including Einstein's) are driven by bulk terms in addition to boundary terms in the constraint evolution equations. In this paper we demonstrate that such bulk generated constraint violations cannot be controlled effectively through the use of boundary conditions alone. Alternative methods of controlling the growth of constraints are still required in such systems.

The most widely used method of controlling the growth of constraints in the Einstein evolution system is called fully constrained evolution. In this method, which is often applied to spherical or axisymmetric problems, symmetry considerations are used to separate the dynamical fields into those that are determined by solving evolution equations and those that are determined by enforcing the constraints at each time step [17-23]. In 3D problems without symmetry there is no obvious way to perform such a separation in a general coordinate system; however, fully constrained 3D methods based on spherical coordinates have yielded promising results [24]. Various groups have studied a closely related method, constraint projection, which can be used for general 3D evolutions in any coordinate system. The idea is to use the evolution system to advance all of the dynamical fields in time, and then at each time step (or whenever the constraints become too large) to force the solution back onto the constraint submanifold by solving the constraint equations (for the conformal factor and the longitudinal part of the extrinsic curvature in the case of the Einstein system). The first preliminary results obtained with this constraint projection technique have been moderately successful [24-26]. Constraint projection has not gained widespread use in 3D simulations, however, due in part to the traditionally high cost of solving the elliptic constraint equations. Difficult questions also remain unanswered about the proper boundary conditions to impose on the constraint equations, for example, at black hole excision boundaries. Moreover, little attention has been 
given to the question of whether these projections correctly map a field configuration onto (or near) the correct point of the constraint submanifold, i.e. the point through which the exact evolution of the system would pass at that time. In particular, it is not clear whether the overall time evolution scheme-including the projections-remains consistent, stable, and convergent.

The need to enforce constraints is a common feature of many problems in mathematical physics besides numerical relativity, and for many problems successful techniques have been developed to ensure that numerical solutions satisfy the needed constraints. Under mild assumptions on the constraints, the subset of the field space satisfying the constraint equations defines a formal differentiable manifold (a classical result due to Ljusternik [27]), and the evolution of a dynamical system of ordinary (ODE) or partial differential equations (PDE) subject to constraints may be viewed as evolution on this submanifold. Constraint control methods for such systems are generally based on ideas from variational mechanics, where the Lagrangian (whose stationary points describe the physical states of the system) is augmented with a sum of terms consisting of products of Lagrange multipliers and the constraints [28-30]. A necessary condition for a configuration point to be a solution of both the field equations and the constraint equations is that the augmented Lagrangian be stationary with respect to variations in both the fundamental fields and the Lagrange multipliers [27]. The additional terms in the augmented Lagrangian involving Lagrange multipliers can be viewed as forcing the dynamics to remain on the constraint submanifold.

These augmented Lagrangian techniques are the basis of well-studied numerical methods for controlling constraint violations in ODE systems. Many ODE systems are subject to algebraic constraints which must be preserved as the solution evolves. For such systems there exist numerical integration techniques that enforce these algebraic constraints exactly, and that also conserve various important properties of the ODE solution (e.g. timereversibility and symplectic structure). These numerical techniques are derived by adding to the ODEs terms chosen to make a suitable augmented Lagrangian for the system stationary [31-35]. The resulting numerical schemes, referred to as "step-and-project" methods, can be thought of as standard time integration steps followed by projections. First a preliminary step is taken forward in time using a standard numerical scheme, after which the solution will generally not satisfy the constraint equations. Then the solution from the preliminary step is corrected using a formal (optimal, or nearest point) projection back onto the constraint submanifold. This projection step typically involves solving algebraic equations. Unlike the simple constraint projection methods used so far in numerical relativity, "step-and-project" numerical methods for constrained systems are wellstudied and well understood. It has been shown that they retain the consistency and stability properties of the original one-step method on which they are based, and they generally have the same convergence properties [35]. These techniques are immediately applicable to constrained PDE systems that are discretized in space to produce constrained ODE systems (as we do, see Sec. IV); and numerical methods based on augmented Lagrangians for PDE systems have also been developed [36,37].

In this paper we apply these augmented variational techniques to obtain equations that project solutions of constrained hyperbolic evolution systems onto the constraint submanifold of the appropriate dynamical field space. We construct projections that are optimal, in the sense that they map a given field configuration to the "nearest" point on the constraint submanifold. We use the natural metric, the symmetrizer, that exists in any symmetric-hyperbolic evolution system to define distances on the space of fields. Hence this optimal projection is the one that minimizes this symmetrizer distance (typically called the energy) between a given field configuration and its projection. The general formalism for constructing such optimal projections for constrained hyperbolic evolution systems is described in Sec. II.

We illustrate these optimal constraint projection ideas in Sec. III by deriving the optimal projection for a new symmetric-hyperbolic representation of the scalar field equation on a fixed background spacetime. This scalar field system has the interesting property that it suffers from constraint violations driven both by bulk terms as well as boundary flux terms in the equations. (And so this system serves as a good model of the pathologies present in the Einstein system.) The optimal projection for this scalar field system is determined by solving a certain elliptic PDE. In Sec. IV we test these optimal projection techniques by studying numerical solutions to this scalar field system on a fixed black hole background spacetime. In particular we demonstrate that constraint-preserving boundary conditions are necessary, but not sufficient, to control the growth of constraints in this pathological scalar field system. We demonstrate that constraint projection succeeds in producing convergent constraintsatisfying solutions, but only if constraint-preserving boundary conditions are used as well. These tests also illustrate that the projections are best performed at fixed time intervals $(\Delta T \approx 2 M$ for this problem) rather than after each time step. And we show that the computational cost of solving the constraint projection equations for this system (using our spectral elliptic solver [38]) is a very small fraction (below $1 \%$ for the resolution needed to achieve roundoff-level accuracy) of the total computational cost of evolving this system. The symmetrizer metric for this model scalar field system (like many 
hyperbolic evolution systems) is not unique; so the projections defined in terms of the symmetrizer are not unique. Nevertheless, we demonstrate for the model scalar field system that numerical evolutions based on these different projections all converge to the same solution. The rate of this convergence is not the same for all projections, however, and we find an "optimal" projection for this system that maximizes this convergence rate.

\section{OPTIMAL CONSTRAINT PROJECTION}

Our objective is to construct a projection operator that maps a given field configuration to the nearest constraintsatisfying configuration (the nearest point on the constraint submanifold). That is, we wish to map an initial point $\bar{u}^{\alpha}$ in the field configuration space to a new point $u^{\alpha}$ that satisfies a set of constraint equations:

$$
c^{A}\left(u^{\alpha}\right)=0 .
$$

(We use Greek indices to label individual components of the dynamical fields, and upper case Latin indices to label the individual components of the constraints.) To find the optimal projection we also need to have a distance measure between field points. We define the needed measure in terms of a symmetric positive-definite metric, $S_{\alpha \beta}$, on the dynamical field space. The distance between field points is then defined as

$$
\|\delta u\|^{2}=\int S_{\alpha \beta}\left(u^{\alpha}-\bar{u}^{\alpha}\right)\left(u^{\beta}-\bar{u}^{\beta}\right) d^{3} x .
$$

Building on the augmented variational techniques commonly used to construct step-and-project constraint control schemes in other areas of numerical analysis $[31,33,35]$, we are now prepared to construct the optimal projection map. We introduce a Lagrangian density $\mathcal{L}$ that consists of the distance between the given field configuration $\bar{u}^{\alpha}$ and its projection $u^{\alpha}$, plus the products of the constraints with Lagrange multipliers. Thus we introduce the Lagrangian density,

$$
\mathcal{L}=S_{\alpha \beta}\left(u^{\alpha}-\bar{u}^{\alpha}\right)\left(u^{\beta}-\bar{u}^{\beta}\right)+\lambda_{A} c^{A} .
$$

The stationarity of the Lagrangian (the volume integral of this Lagrangian density) with respect to variations of the Lagrange multipliers $\lambda_{A}$ enforces the constraints, while stationarity with respect to variations of the fields $u^{\alpha}$ are necessary conditions for the projection to minimize the distance to the constraint submanifold.

The optimal projection procedure outlined above could be carried out using any definition of the distance between field points, e.g., using any positive-definite metric $S_{\alpha \beta}$ on the space of fields. For a particular problem this distance measure should be chosen to be the natural measure associated with that problem. Our primary interest here is the construction of projections for constrained hyperbolic evolution systems. So we will focus our attention on fields $u^{\alpha}$ that satisfy a first-order evolu- tion equation of the form

$$
\partial_{t} u^{\alpha}+A_{\beta}^{k \alpha} \partial_{k} u^{\beta}=F^{\alpha} .
$$

We use lower case Latin indices to label spatial coordinates $x^{k}, \partial_{t}=\partial / \partial t$ to denote time derivatives, and $\partial_{k}=$ $\partial / \partial x^{k}$ to denote spatial derivatives. Such systems are called symmetric-hyperbolic if they have a positivedefinite metric $S_{\alpha \beta}$ on the space of fields (typically called the symmetrizer) that symmetrizes the characteristic matrices:

$$
S_{\alpha \gamma} A_{\beta}^{k \gamma} \equiv A_{\alpha \beta}^{k}=A_{\beta \alpha}^{k} .
$$

The well-posedness of the initial value problem for linear symmetric-hyperbolic evolution systems is demonstrated by establishing bounds on the square-integral norm of the dynamical fields defined with this symmetrizer metric $[39,40]$. This metric defines the meaningful measure on the dynamical field space for symmetric-hyperbolic systems, so this is the appropriate measure to use for constructing optimal constraint projections for these systems. Most hyperbolic evolution systems of interest in mathematical physics (including many representations of the Einstein system) are symmetric-hyperbolic, and so we limit our consideration here to systems of this type.

In Sec. III we use the procedure outlined above to construct explicitly the optimal projection for the relatively simple case of the scalar wave equation on a curved background spacetime. But before we focus on that special case, we take a few (rather more abstract) steps in the construction of this projection for the general case. To do this we assume that the constraints $c^{A}$ are linear in the derivatives of the dynamical fields:

$$
c^{A}=K_{\beta}^{k A} \partial_{k} u^{\beta}+L^{A},
$$

where $K_{\beta}^{k A}$ and $L^{A}$ may depend on $u^{\alpha}$ but not its derivatives. The constraints have this general form in many evolution systems of interest (e.g., the Einstein system, the Maxwell system, the incompressible fluid system). In this case we can explicitly compute the variations of the Lagrangian density defined in Eq. (3):

$$
\begin{aligned}
\frac{\delta \mathcal{L}}{\delta u^{\alpha}} \delta u^{\alpha}= & \delta u^{\alpha}\left\{2 S_{\alpha \beta}\left(u^{\beta}-\bar{u}^{\beta}\right)-\partial_{k}\left(\lambda_{A} K^{k A}{ }_{\alpha}\right)\right. \\
+ & \left.\lambda_{A}\left(\partial_{\alpha} K^{k A}{ }_{\beta} \partial_{k} u^{\beta}+\partial_{\alpha} L^{A}\right)\right\} \\
+ & \partial_{k}\left(\lambda_{A} K^{k A}{ }_{\alpha} \delta u^{\alpha}\right), \\
& \frac{\delta \mathcal{L}}{\delta \lambda_{A}} \delta \lambda_{A}=c^{A} \delta \lambda_{A} .
\end{aligned}
$$

Here we use the notation $\partial_{\alpha} \equiv \partial / \partial u^{\alpha}$ to denote derivatives with respect to the fields. We have also assumed that the symmetrizer $S_{\alpha \beta}$ may depend on $\bar{u}^{\alpha}$ but not $u^{\alpha}$. We wish to find the stationary points of this Lagrangian with respect to arbitrary variations in the fields $u^{\alpha}$ and the Lagrange multipliers $\lambda_{A}$. Stationarity with respect to the 
variations of these quantities (that vanish on the boundaries) implies that

$$
\begin{aligned}
& 0= u^{\alpha}-\bar{u}^{\alpha}-\frac{1}{2} S^{\alpha \beta} \partial_{k}\left(\lambda_{A} K_{\beta}^{k A}\right) \\
&+ \frac{1}{2} \lambda_{A} S^{\alpha \beta}\left(\partial_{\beta} K^{k A}{ }_{\gamma} \partial_{k} u^{\gamma}+\partial_{\beta} L^{A}\right), \\
& 0=c^{A}=K^{k A}{ }_{\beta} \partial_{k} u^{\beta}+L^{A}
\end{aligned}
$$

at each interior point, and stationarity with respect to boundary variations implies that

$$
0=n_{k} \lambda_{A} K_{\beta}^{k A}
$$

at each boundary point, where $n_{k}$ is the outward directed unit normal to the surface. We use the notation $S^{\alpha \beta}$ to denote the inverse of $S_{\alpha \beta}$. The general idea is to use Eqs. (9) and (10), with appropriate boundary conditions [such as those provided by Eq. (11)], to determine the field configuration $u^{\beta}$ and the Lagrange multipliers $\lambda_{A}$ for any given field point $\bar{u}^{\alpha}$. If $u^{\alpha}$ and $\lambda_{A}$ satisfying these equations can be found, then we are guaranteed that the field $u^{\alpha}$ is the constraint-satisfying solution nearest the point $\bar{u}^{\alpha}$ as desired. We do not know whether these equations always admit solutions in the general case. So in Sec. III we study in detail this optimal projection for the simple case of the scalar field equations on a fixed background spacetime. We show that solutions to the optimal projection equations always exist and are relatively easy to compute numerically in this simple case. And in Sec. IV we show that this optimal projection is very effective in controlling the growth of constraints for the scalar field system.

\section{SCALAR FIELDS IN CURVED SPACETIME}

In this section we examine in some detail the scalar wave system on a fixed background spacetime. In Sec. III A we review the standard treatment of this system, and then modify it so that it exhibits bulk generated constraint violations in addition to the boundary generated violations present in the standard system. This new, more pathological, symmetric-hyperbolic scalar field system now serves as a good model of the constraintviolating problems inherent in the Einstein system. We construct constraint-preserving boundary conditions for this system in Sec. III B, and the optimal projection map for this system in Sec. IIIC following the procedure outlined in Sec. II.

\section{A. Modified scalar wave system}

The standard scalar field equation on a fixed background spacetime is

$$
\nabla^{\mu} \nabla_{\mu} \psi=0
$$

where $\psi$ represents the scalar field and $\nabla_{\mu}$ the covariant derivative associated with the background spacetime metric. We represent the background spacetime metric in terms of the usual $3+1$ splitting:

$$
d s^{2}=-N^{2} d t^{2}+g_{i j}\left(d x^{i}+N^{i} d t\right)\left(d x^{j}+N^{j} d t\right),
$$

where the lapse $N$ and the spatial metric $g_{i j}$ are assumed to be positive-definite, while the shift $N^{i}$ is arbitrary. The equation for the scalar field $\psi$, Eq. (12), can be reexpressed as a first-order evolution system in the standard way (see, e.g., Ref. [41]):

$$
\begin{gathered}
\partial_{t} \psi-N^{k} \partial_{k} \psi=-N \Pi, \\
\partial_{t} \Pi-N^{k} \partial_{k} \Pi+N g^{k i} \partial_{k} \Phi_{i}=N J^{i} \Phi_{i}+N K \Pi, \\
\partial_{t} \Phi_{i}-N^{k} \partial_{k} \Phi_{i}+N \partial_{i} \Pi=-\Pi \partial_{i} N+\Phi_{j} \partial_{i} N^{j} .
\end{gathered}
$$

The field $\Phi_{i}$ represents the spatial gradient $\partial_{i} \psi$, and $\Pi$ represents the time-derivative of $\psi$ [and is defined precisely by Eq. (14)]. The auxiliary quantities $K$ (the trace of the extrinsic curvature) and $J^{i}$ depend only on the background spacetime geometry, and are defined by

$$
\begin{gathered}
J^{i}=-N^{-1} g^{-1 / 2} \partial_{j}\left(N g^{1 / 2} g^{i j}\right), \\
K=-N^{-1} g^{-1 / 2}\left[\partial_{t} g^{1 / 2}-\partial_{j}\left(g^{1 / 2} N^{j}\right)\right] .
\end{gathered}
$$

Solutions to the first-order system, Eqs. (14)-(16), are also solutions to Eq. (12) only if the constraints are satisfied: $0=c^{A} \equiv\left\{C_{i}, C_{i j}\right\}$, where

$$
\begin{gathered}
C_{i}=\partial_{i} \psi-\Phi_{i}, \\
C_{i j}=\partial_{[i} \Phi_{j]} .
\end{gathered}
$$

Although the second constraint, $C_{i j}=0$, follows from the first, $C_{i}=0$, the converse is not true. Hence we include both constraints in the analysis here. Note that both constraints are necessary to construct a first-order hyperbolic evolution system for the constraint quantities [discussed below, Eqs. (29) and (30)]. Note also that the analogues of both constraints play essential roles in first-order hyperbolic formulations of Einstein's equations.

We now generalize the evolution system, Eqs. (14)(16), somewhat by adding multiples of the constraint $C_{i}$ to Eqs. (14) and (16):

$$
\begin{gathered}
\partial_{t} \psi-N^{k} \partial_{k} \psi=-N \Pi+\gamma_{1} N^{k} C_{k}, \\
\partial_{t} \Phi_{i}-N^{k} \partial_{k} \Phi_{i}+N \partial_{i} \Pi= \\
-\Pi \partial_{i} N+\Phi_{j} \partial_{i} N^{j} \\
+\gamma_{2} N C_{i},
\end{gathered}
$$

where $\gamma_{1}$ and $\gamma_{2}$ are arbitrary constants. The constraintsatisfying solutions to these equations are the same as those of the original system; but as we shall see, the constraint-violating properties of the new system are 
significantly different from those of the original. Substituting the definition of $C_{i}$ in Eqs. (21) and (22) gives us new evolution equations for $\psi$ and $\Phi_{i}$ :

$$
\begin{gathered}
\partial_{t} \psi-\left(1+\gamma_{1}\right) N^{k} \partial_{k} \psi=-N \Pi-\gamma_{1} N^{k} \Phi_{k}, \\
\partial_{t} \Phi_{i}-N^{k} \partial_{k} \Phi_{i}+N \partial_{i} \Pi-\gamma_{2} N \partial_{i} \psi= \\
-\Pi \partial_{i} N+\Phi_{j} \partial_{i} N^{j}-\gamma_{2} N \Phi_{i} .
\end{gathered}
$$

The first-order system that represents the scalar wave equation, Eqs. (15), (23), and (24), has the standard firstorder form,

$$
\partial_{t} u^{\alpha}+A_{\beta}^{k \alpha} \partial_{k} u^{\beta}=F^{\alpha},
$$

where $u^{\alpha}=\left\{\psi, \Pi, \Phi_{i}\right\}$. Systems of this type are called symmetric-hyperbolic if there exists a symmetric positive-definite tensor $S_{\alpha \beta}$ on the space of fields that symmetrizes the characteristic matrices $A^{k \alpha}{ }_{\beta}$ :

$$
S_{\alpha \gamma} A_{\beta}^{k \gamma} \equiv A_{\alpha \beta}^{k}=A_{\beta \alpha}^{k} .
$$

The most general symmetrizer for our new scalar wave system is (up to an overall factor),

$$
\begin{aligned}
d s^{2} & =S_{\alpha \beta} d u^{\alpha} d u^{\beta}, \\
& =\Lambda^{2} d \psi^{2}-2 \gamma_{2} d \psi d \Pi+d \Pi^{2}+g^{i j} d \Phi_{i} d \Phi_{j},
\end{aligned}
$$

where $\Lambda$ is an arbitrary nonvanishing function. This $S_{\alpha \beta}$ symmetrizes the characteristic matrices $A_{\alpha \beta}^{k}$ so long as $\gamma_{1} \gamma_{2}=0$. Thus we must take at least one of these parameters to be zero for our new system to be symmetric-hyperbolic. This symmetrizer is positivedefinite whenever

$$
\Lambda^{2}>\gamma_{2}^{2} \text {. }
$$

In this case $S_{\alpha \beta}$ provides a dynamically meaningful measure of the distance between field configurations, which we use to define our optimal constraint projection operator in Sec. IIIC.

The evolution of the constraints follows from the principal evolution system, Eqs. (15), (23), and (24),:

$$
\begin{gathered}
\partial_{t} C_{i}-\left(1+\gamma_{1}\right) \mathcal{L}_{\vec{N}} C_{i}=2 \gamma_{1} N^{j} C_{j i}-\gamma_{2} N C_{i}, \\
\partial_{t} C_{i j}-\mathcal{L}_{\vec{N}} C_{i j}=-\gamma_{2} N C_{i j}-\gamma_{2} C_{[i} \partial_{j]} N,
\end{gathered}
$$

where $\mathcal{L}_{\vec{N}}$ represents the Lie derivative along the shift vector $N^{i}$. If the constraints are satisfied at some initial time, then these equations guarantee (at least at the analytical level) that the constraints remain satisfied in the domain of dependence of the initial data. These equations also show that any constraint violations in this system will be advected along a congruence of timelike curves. Constraint violations can therefore flow into the computational domain if these curves intersect the boundaries. And like the Einstein evolution system, these equations also contain bulk terms that amplify any ex- isting constraint violations. When $\gamma_{1}=0$ we see that Eq. (29) implies that the constraint $C_{i}$ has the simple time dependence $C_{i}(\tau)=C_{i}(0) e^{-\gamma_{2} \tau}$, where $\tau$ measures proper time as seen by a hypersurface orthogonal observer. Whenever $\gamma_{2}<0$ this constraint grows exponentially, and in this case the modified scalar wave system serves as a good model of the constraint violations in the Einstein system. (Constraint violations of all wavelengths grow exponentially in this system, and so it may be even more pathological than the Einstein system where constraint-violating instabilities are typically dominated by long wavelength modes [2,3].) Conversely, if $\gamma_{2}>0$ then this modified scalar wave system exponentially suppresses any residual constraint violations that may be present in the initial data. This latter property suggests that analogous terms could be introduced to control some of the bulk constraint-violating terms in the Einstein system.

\section{B. Constraint-preserving boundary conditions}

Boundary conditions for hyperbolic evolution systems are defined in terms of the characteristic fields of these systems, so we must construct these fields for our modified scalar wave system. The characteristic fields are defined with respect to a spatial direction at each point, represented here by a unit normal covector field $n_{k}$. For the purposes of imposing boundary conditions, the appropriate $n_{k}$ is the outward-pointing normal to the boundary. Given a direction field $n_{k}$ we define the left eigenvectors $e^{\hat{\alpha}}{ }_{\alpha}$ of the characteristic matrix $n_{k} A^{k \alpha}{ }_{\beta}$ by

$$
e_{\alpha}^{\hat{\alpha}} n_{k} A_{\beta}^{k \alpha}=v_{(\hat{\alpha})} e_{\beta}^{\hat{\alpha}},
$$

where $v_{(\hat{\alpha})}$ denotes the eigenvalue (also called the characteristic speed). The index $\hat{\alpha}$ labels the various eigenvectors and eigenvalues, and there is no summation over $\hat{\alpha}$ in Eq. (31). Since we are interested in hyperbolic evolution systems, the space of eigenvectors has the same dimension as the space of dynamical fields, and the matrix $e^{\hat{\alpha}}{ }_{\beta}$ is invertible. The projections of the dynamical fields $u^{\alpha}$ onto these left eigenvectors are called the characteristic fields $u^{\hat{\alpha}}$ :

$$
u^{\hat{\alpha}}=e_{\beta}^{\hat{\alpha}} u^{\beta} .
$$

At each boundary point, boundary conditions must be imposed on any characteristic field having negative characteristic speed, $v_{(\hat{\alpha})}<0$, at that point $[42,43]$. We refer to fields with $\boldsymbol{v}_{(\hat{\alpha})}<0$ as the incoming characteristic fields at that point. Conversely, those characteristic fields having non-negative characteristic speeds (the outgoing fields) must not have boundary conditions imposed on them there.

The characteristic fields for the symmetric-hyperbolic representations $\left(\gamma_{1} \gamma_{2}=0\right)$ of the scalar wave system are the quantities $u^{\hat{\alpha}}=\left\{Z^{1}, Z_{i}^{2}, U^{1 \pm}\right\}$ : 


$$
\begin{gathered}
Z^{1}=\psi, \\
Z_{i}^{2}=P_{i}^{k} \Phi_{k}, \\
U^{1 \pm}=\Pi \pm n^{k} \Phi_{k}-\gamma_{2} \psi,
\end{gathered}
$$

where $P^{k}{ }_{i}=\delta^{k}{ }_{i}-n^{k} n_{i}, n^{k}=g^{k j} n_{j}$, and $n^{k} n_{k}=1$. The fundamental fields $u^{\alpha}$ can be reconstructed from the characteristic fields $u^{\hat{\alpha}}$ by inverting Eq. (32):

$$
\begin{gathered}
\psi=Z^{1}, \\
\Pi=\frac{1}{2}\left(U^{1+}+U^{1-}\right)+\gamma_{2} Z^{1}, \\
\Phi_{i}=\frac{1}{2}\left(U^{1+}-U^{1-}\right) n_{i}+Z_{i}^{2} .
\end{gathered}
$$

The characteristic field $Z^{1}$ propagates with speed $-\gamma_{1} n_{k} N^{k} / N$, the field $Z_{i}^{2}$ with speed 0 , and the fields $U^{1 \pm}$ with speeds \pm 1 relative to the hypersurface orthogonal observers. The coordinate characteristic speeds of these fields are $-\left(1+\gamma_{1}\right) n_{k} N^{k},-n_{k} N^{k}$ and $-n_{k} N^{k} \pm$ $N$, respectively.

At each boundary point, boundary conditions are required on each characteristic field whose coordinate characteristic speed is negative at that point. The field $U^{1-}$, in particular, requires a boundary condition on all timelike boundaries. For the standard representation of the scalar field system, Eqs. (14)-(16), the boundary condition $U^{1-}=\Pi-n^{k} \Phi_{k}=0$ is used to ensure (approximately) that no scalar waves enter the computational domain. We wish to enforce this condition on our generalized scalar field system, Eqs. (15), (23), and (24), in such a way that the physical (constraint-satisfying) solutions are the same for all values of the parameters $\gamma_{1}$ and $\gamma_{2}$. Since $U^{1-}$ depends on $\gamma_{2}$, Eq. (35), the proper boundary condition must also depend on $\gamma_{2}: U^{1-}+\gamma_{2} \psi=\Pi-n^{k} \Phi_{k}=0$. Thus the appropriate boundary condition to impose on $U^{1-}$ is $U^{1-}=-\gamma_{2} \psi$. The freezing form of this boundary condition (as used in our code) is,

$$
\partial_{t} U^{1-}=-\gamma_{2} \partial_{t} \psi
$$

For boundary conditions on the fields $Z^{1}$ and $Z_{i}^{2}$ (when necessary), we explore two choices: One is the freezing boundary condition $\partial_{t} Z^{i}=\partial_{t} Z_{i}^{2}=0$. In Sec. IV we show that this boundary condition allows constraint violations to enter the computational domain through the boundaries. Therefore, we also explore conditions that prevent this influx of constraint violations: When the fields $Z^{1}$ and/or $Z_{i}^{2}$ require boundary conditions, we set

$$
\begin{gathered}
\partial_{t} Z^{1}=N^{k} \Phi_{k}-N \Pi, \\
\partial_{t} Z_{i}^{2}=P^{k}{ }_{i} \partial_{k} \partial_{t} \psi .
\end{gathered}
$$

Eq. (40) is based on Eq. (14) combined with Eq. (19), while Eq. (41) is derived from the time-derivative of Eq. (19). We note that with the choice $\gamma_{1}=-1$, the field $Z^{1}$ never requires a boundary condition. We also note that the term $\partial_{t} \psi$ that appears on right side of Eqs. (39) and (41) must be evaluated using the appropriate expression for $\partial_{t} \psi=\partial_{t} Z^{1}$ on this boundary: Eq. (40) when $Z^{1}$ requires a boundary condition, or Eq. (23) when no boundary condition is required. In Sec. IV we compare numerically the results of using these constraintpreserving boundary conditions with the use of the freezing boundary conditions $\partial_{t} Z^{1}=\partial_{t} Z_{i}^{2}=0$ on these fields.

\section{Optimal constraint projection}

The idea is to use the full evolution system, Eqs. (15), (23), and (24), to evolve initial data forward in time an amount $\Delta T$ and then (when the constraint violations become too large) to project this solution back onto the constraint submanifold in some optimal way. Let $\bar{u}^{\alpha}=$ $\left\{\bar{\psi}, \bar{\Pi}, \bar{\Phi}_{i}\right\}$ denote the solution obtained directly from this free evolution step. This solution $\bar{u}^{\alpha}$ may not satisfy the constraints because roundoff or truncation level constraint violations have been amplified, or constraint violations have flowed through the boundaries. Thus we wish to project $\bar{u}^{\alpha}$ in an optimal way back onto the constraint submanifold. Following the procedure outlined in Sec. II we construct a Lagrangian density,

$$
\begin{aligned}
\mathcal{L}= & g^{1 / 2}\left[S_{\alpha \beta}\left(u^{\alpha}-\bar{u}^{\alpha}\right)\left(u^{\beta}-\bar{u}^{\beta}\right)+\lambda_{A} c^{A}\right] \\
= & g^{1 / 2}\left[\Lambda^{2}(\psi-\bar{\psi})^{2}-2 \gamma_{2}(\psi-\bar{\psi})(\Pi-\bar{\Pi})\right. \\
& +(\Pi-\bar{\Pi})^{2}+g^{i j}\left(\Phi_{i}-\bar{\Phi}_{i}\right)\left(\Phi_{j}-\bar{\Phi}_{j}\right) \\
& \left.+\lambda^{i}\left(\partial_{i} \psi-\Phi_{i}\right)+\lambda^{i j} \partial_{[i} \Phi_{j]}\right],
\end{aligned}
$$

using the symmetrizer $S_{\alpha \beta}$ of the hyperbolic evolution system, Eq. (27), and the Lagrange multipliers $\lambda_{A}=$ $\left\{\lambda^{i}, \lambda^{i j}\right\}$. The stationary points of the Lagrangian,

$$
L=\int \mathcal{L} d^{3} x,
$$

with respect to variations in $u^{\alpha}$ and $\lambda_{A}$ define the optimally projected field configuration $u^{\alpha}$. We have included the multiplicative factor $g^{1 / 2}=\left(\operatorname{det} g_{i j}\right)^{1 / 2}$ in Eq. (42) to ensure that $L$ is coordinate-invariant.

The scalar field constraint Lagrangian density, Eq. (42), has the following variations:

$$
\begin{aligned}
\frac{\delta \mathcal{L}}{\delta \psi} \delta \psi= & 2 g^{1 / 2}\left[\Lambda^{2}(\psi-\bar{\psi})-\gamma_{2}(\Pi-\bar{\Pi})\right] \delta \psi \\
& -\partial_{i}\left(g^{1 / 2} \lambda^{i}\right) \delta \psi+\partial_{i}\left(g^{1 / 2} \lambda^{i} \delta \psi\right),
\end{aligned}
$$

$$
\frac{\delta \mathcal{L}}{\delta \Pi} \delta \Pi=2 g^{1 / 2}\left[\Pi-\bar{\Pi}-\gamma_{2}(\psi-\bar{\psi})\right] \delta \Pi,
$$




$$
\begin{aligned}
\frac{\delta \mathcal{L}}{\delta \Phi_{i}} \delta \Phi_{i}= & {\left[2 g^{1 / 2} g^{i j}\left(\Phi_{i}-\bar{\Phi}_{i}\right)-g^{1 / 2} \lambda^{j}\right.} \\
& \left.-\partial_{i}\left(g^{1 / 2} \lambda^{i j}\right)\right] \delta \Phi_{j}+\partial_{i}\left(g^{1 / 2} \lambda^{i j} \delta \Phi_{j}\right) \\
& \frac{\delta \mathcal{L}}{\delta \lambda^{i}} \delta \lambda^{i}=g^{1 / 2}\left(\partial_{i} \psi-\Phi_{i}\right) \delta \lambda^{i} \\
& \frac{\delta \mathcal{L}}{\delta \lambda^{i j}} \delta \lambda^{i j}=g^{1 / 2} \partial_{[i} \Phi_{j]} \delta \lambda^{i j}
\end{aligned}
$$

We require that the Lagrangian $L$ from Eq. (43) be stationary with respect to all variations in the dynamical fields $\delta u^{\alpha}=\left\{\delta \psi, \delta \Pi, \delta \Phi_{i}\right\}$ (including those that do not vanish on the boundaries) as well as all variations in the Lagrange multipliers $\delta \lambda_{A}=\left\{\delta \lambda^{i}, \delta \lambda^{i j}\right\}$. From Eqs. (44)(46), it follows that

$$
\begin{gathered}
\psi=\bar{\psi}+\gamma_{2} \Lambda^{-2}(\Pi-\bar{\Pi})+\frac{1}{2} \Lambda^{-2} g^{-1 / 2} \partial_{i}\left(g^{1 / 2} \lambda^{i}\right), \\
\Pi=\bar{\Pi}+\gamma_{2}(\psi-\bar{\psi}), \\
\Phi_{i}=\bar{\Phi}_{i}+\frac{1}{2} g_{i j} \lambda^{j}+\frac{1}{2} g^{-1 / 2} g_{i j} \partial_{k}\left(g^{1 / 2} \lambda^{k j}\right),
\end{gathered}
$$

and Eqs. (47) and (48) imply that the projected solution satisfies the constraints. We now solve Eq. (51) for $\lambda^{i}$, substitute it into Eq. (49), and use Eqs. (47) and (50), to obtain the following equation for $\psi$,

$$
\nabla^{i} \nabla_{i} \psi-\left(\Lambda^{2}-\gamma_{2}^{2}\right) \psi=\nabla^{i} \bar{\Phi}_{i}-\left(\Lambda^{2}-\gamma_{2}^{2}\right) \bar{\psi},
$$

where $\nabla_{i}$ represents the spatial covariant derivative that is compatible with $g_{i j}$. In deriving this equation we have also used the fact that the term $\partial_{i} \partial_{k}\left(g^{1 / 2} \lambda^{k i}\right)$ vanishes identically because $\lambda^{i j}$ is antisymmetric. Equation (52) is just the covariant inhomogeneous Helmholtz equation. We note that the parameters must satisfy the condition $\Lambda^{2}-\gamma_{2}^{2}>0$ for the evolution system to be symmetrichyperbolic. Solving Eq. (52) determines the optimal projection $\psi$; the optimal $\Pi$ is determined from Eq. (50),

$$
\Pi=\bar{\Pi}+\gamma_{2}(\psi-\bar{\psi}) ;
$$

and the optimal $\Phi_{i}$ is obtained by enforcing the constraint,

$$
\Phi_{i}=\partial_{i} \psi
$$

We note that the Lagrange multiplier $\lambda^{i j}$ does not play any essential role in this analysis: we could just as well have set $\lambda^{i j}=0$ and still obtained the same projection. This makes sense, because the constraint $C_{i j}$ is really a consequence of the constraint $C_{i}$ in this case.

The evolution equations for $\Pi$ and $\Phi_{i}$, Eqs. (15) and (16), decouple from the larger scalar field evolution system, Eqs. (15), (23), and (24), when $\gamma_{2}=0$. It is some- times of interest to consider the properties of this smaller system, Eqs. (15) and (16), subject to the single constraint, Eq. (20). The optimal constraint projection for this reduced system consists of Eqs. (50) and (51) (with $\lambda^{i}=\gamma_{2}=0$ ), together with the single constraint equation $\partial_{[i} \Phi_{j]}=0$. This constraint equation implies that $\Phi_{i}=\partial_{i} \psi$ for some scalar function $\psi$. Inserting this expression for $\Phi_{i}$ in Eq. (51), multiplying by $g^{1 / 2} g^{i j}$, and taking the divergence, we obtain the following equation for $\psi$,

$$
\nabla^{i} \nabla_{i} \psi=\nabla^{i} \bar{\Phi}_{i}
$$

In deriving this equation we have used the fact that the term $\partial_{i} \partial_{k}\left(g^{1 / 2} \lambda^{k i}\right)$ vanishes identically because $\lambda^{i j}$ is antisymmetric. The optimal projection in this reduced system then sets $\Pi=\bar{\Pi}$ and $\Phi_{i}=\partial_{i} \psi$, where $\psi$ is the solution to Eq. (55). We note that Eq. (55) is just the $\Lambda^{2}-$ $\gamma_{2}^{2}=0$ limit of the original projection Eq. (52).

Unfortunately the optimal constraint projection for the scalar field system is not unique, because the parameter $\Lambda$ in the symmetrizer metric is not unique. We have seen that taking the limit $\Lambda^{2} \rightarrow \gamma_{2}^{2}$ is equivalent to ignoring the evolution of the scalar field $\bar{\psi}$ in constructing the optimal projection. Alternatively, the limit $\Lambda \rightarrow \infty$ corresponds to the simple projection $\psi=\bar{\psi}, \Pi=\bar{\Pi}$, and $\Phi_{i}=\partial_{i} \bar{\psi}$. In this limit, no elliptic equation has to be solved, and the evolution of the field $\bar{\Phi}_{i}$ is effectively ignored when constructing the projection. We expect that the optimal choice of $\Lambda$ will be one for which $1 / \Lambda$ corresponds to some characteristic length or time-scale associated with the particular problem. We explore in Sec. IV C the properties of these projection operators for a range of $\Lambda$, and show that an optimal value does exist. When $\gamma_{2} \neq 0$ the optimal choice seems to be $\Lambda^{2}=2 \gamma_{2}^{2}$, where $1 /\left|\gamma_{2}\right|$ is the time-scale on which the constraints are amplified.

Finally, we must consider the boundary conditions for the projection equations that determine $\psi$, i.e., Eq. (52) or (55). In general, boundary conditions for the projection equations must satisfy two criteria: First, they must be consistent with boundary conditions imposed on the evolution equations, and second, the projection equations plus boundary conditions must not modify solutions that already satisfy the constraints. Typically, we enforce approximate outgoing wave boundary conditions on the evolution equations. For the case of the scalar wave equation, the approximate outgoing wave boundary condition, Eq. (39), sets $U^{1-}=-\gamma_{2} \psi$ or equivalently $n^{k} \Phi_{k}=\Pi$ on the boundaries (where $n^{k}$ is the outward directed unit normal). Since $\Phi_{i}=\partial_{i} \psi$ in these projected solutions, the appropriate boundary condition to impose on $\psi$ in Eq. (52) or (55) in this case would be

$$
n^{k} \partial_{k} \psi=\Pi=\bar{\Pi}+\gamma_{2}(\psi-\bar{\psi}) .
$$

Alternatively we can derive boundary conditions for $\psi$ from the requirement that the boundary variations of the 
Lagrangian vanish. The divergence terms in Eqs. (44) and (46) imply that

$$
0=n_{k} \lambda^{k}=n_{k} \lambda^{k i},
$$

on the boundaries for the scalar field system. A short calculation (using the fact that $n_{k}$ is proportional to a gradient, and $\lambda^{k i}$ is antisymmetric) shows that $n_{i} \partial_{k}\left(g^{1 / 2} \lambda^{k i}\right)=0$, so we see from Eq. (51) that the natural boundary condition is

$$
n^{k} \partial_{k} \psi=n^{k} \bar{\Phi}_{k} .
$$

If the approximate outgoing wave boundary condition, $n^{k} \bar{\Phi}_{k}=\bar{\Pi}$, was used in the free evolution step, then the natural boundary condition Eq. (58) differs from Eq. (56) by the term $\gamma_{2}(\psi-\bar{\psi})$. For the constraint projections described in Sec. IV, we impose the Robin boundary condition Eq. (56) on the solutions of Eq. (52) at the boundaries where $U^{1-}$ requires a boundary condition in the evolution step, and Eq. (58) on the solutions at all other boundaries (e.g. inside an event horizon). We note that the discrepancy between the natural and the physical outgoing boundary condition could be eliminated by adding an appropriate boundary term to the constraint projection Lagrangian.

\section{NUMERICAL RESULTS}

We have studied the effectiveness of the optimal constraint projection methods developed in Secs. II and III for the case of a scalar field propagating on a fixed black hole spacetime. For these simulations we use the KerrSchild form of the Schwarzschild metric as our background geometry:

$$
d s^{2}=-d t^{2}+\frac{2 M}{r}(d t+d r)^{2}+d r^{2}+r^{2} d \Omega^{2} .
$$

We express all lengths and times associated with these simulations in units of the mass, $M$, of this black hole. Our computational domain consists of a spherical shell extending from $r_{\min }=1.9 \mathrm{M}$ (just inside the black hole event horizon) to $r_{\max }=11.9 M$. For initial data we use a constraint-satisfying Gaussian shaped pulse with dipolar angular structure,

$$
\begin{gathered}
\psi=0, \\
\Pi=Y_{10}(\theta, \varphi) e^{-\left(r-r_{0}\right)^{2} / w^{2}}, \\
\Phi_{i}=0,
\end{gathered}
$$

with $r_{0}=5 M$ and $w=1 M$. The value of $\Pi$ is about $2 \times$ $10^{-21}$ at the outer boundary of our computational domain, below the level of double precision roundoff error.

For the remainder of this section we describe briefly the numerical methods used to solve this problem. Then in Sec. IVA we describe three numerical simulations designed to explore the effects of boundary conditions on the evolution of the constraints in these solutions. In Sec. IV B we describe two additional numerical simulations that illustrate the effectiveness of constraint projection in controlling the growth of constraints. And finally in Sec. IV C we explore ways to optimize the use of the constraint projection method and measure its computational cost.

All numerical computations presented here are performed using a pseudospectral collocation method. Our numerical methods are essentially the same as those we have applied to evolution problems with the Einstein system [1-3,44], with scalar fields [41], and with the Maxwell system [8]. Given a system of partial differential equations

$$
\partial_{t} u^{\alpha}(\mathbf{x}, t)=\mathcal{F}^{\alpha}\left[u(\mathbf{x}, t), \partial_{i} u(\mathbf{x}, t)\right],
$$

where $u^{\alpha}$ is a collection of dynamical fields, the solution $u^{\alpha}(\mathbf{x}, t)$ is expressed as a time-dependent linear combination of $N$ spatial basis functions $\phi_{k}(\mathbf{x})$ :

$$
u_{N}^{\alpha}(\mathbf{x}, t)=\sum_{k=0}^{N-1} \tilde{u}_{k}^{\alpha}(t) \phi_{k}(\mathbf{x}) .
$$

We expand each scalar function $(\psi$ and $\Pi)$ and the Cartesian components of each vector $\left(\Phi_{x}, \Phi_{y}\right.$, and $\left.\Phi_{z}\right)$ in terms of the basis functions $T_{n}(\rho) Y_{l m}(\theta, \varphi)$, where $Y_{l m}$ are spherical harmonics and $T_{n}(\rho)$ are Chebyshev polynomials with

$$
\rho=\frac{2 r-r_{\max }-r_{\min }}{r_{\max }-r_{\min }} .
$$

We use spherical harmonics with $\ell \leq \ell_{\max }=5$ and a varying number of Chebyshev polynomials with degrees $N_{r} \leq 81$. Spatial derivatives are evaluated analytically using the known derivatives of the basis functions:

$$
\partial_{i} u_{N}^{\alpha}(\mathbf{x}, t)=\sum_{k=0}^{N-1} \tilde{u}_{k}^{\alpha}(t) \partial_{i} \phi_{k}(\mathbf{x})
$$

Associated with the basis functions is a set of $N_{c}$ collocation points $\mathbf{x}_{i}$. Given spectral coefficients $\tilde{u}_{k}^{\alpha}(t)$, the function values at the collocation points $u^{\alpha}\left(\mathbf{x}_{i}, t\right)$ are computed by Eq. (64). Conversely, the spectral coefficients are obtained by the inverse transform

$$
\tilde{u}_{k}^{\alpha}(t)=\sum_{i=0}^{N_{c}-1} w_{i} u_{N}^{\alpha}\left(\mathbf{x}_{i}, t\right) \phi_{k}\left(\mathbf{x}_{i}\right),
$$

where $w_{i}$ are weights specific to the choice of basis functions and collocation points; thus it is straightforward to transform between the spectral coefficients $\tilde{u}_{k}^{\alpha}(t)$ and the function values at the collocation points $u_{N}^{\alpha}\left(\mathbf{x}_{i}, t\right)$. The partial differential equation, Eq. (63), is now rewritten using Eqs. (64)-(67) as a set of ordinary differential equations for the function values at the collocation points, 


$$
\partial_{t} u_{N}^{\alpha}\left(\mathbf{x}_{i}, t\right)=G_{i}^{\alpha}\left[u_{N}\left(\mathbf{x}_{j}, t\right)\right],
$$

where $G_{i}^{\alpha}$ depends on $u_{N}^{\alpha}\left(\mathbf{x}_{j}, t\right)$ for all $j$. This system of ordinary differential equations, Eq. (68), is integrated in time using a fourth-order Runge-Kutta algorithm. Boundary conditions are incorporated into the right side of Eq. (68) using the technique of Bjørhus [45]. The time step is typically chosen to be about one fifth the distance between the closest collocation points, which ensures that the Courant condition is well satisfied. This small time step is needed to reduce the time discretization error to the same order of magnitude as the spatial discretization error at radial resolution $N_{r}=61$.

Elliptic partial differential equations, Eq. (52) or (55), are solved using similar pseudospectral collocation methods. As detailed in Ref. [38], we consider a mixed real/spectral expansion of the desired solution $\psi(\mathbf{x})$ :

$$
\psi\left(\rho_{n}, \theta, \phi\right)=\sum_{l=0}^{l_{\max }} \sum_{m=-l}^{l} \hat{\psi}_{l m n} Y_{l m}(\theta, \phi)
$$

where $\rho_{n}$ (for $n=0, \ldots, N_{r}-1$ ) are the collocation points of the Chebyshev expansion in (rescaled) radius $\rho$. Given a set of coefficients $\hat{\psi}_{l m n}$, we can evaluate the residual of the elliptic equation and the residual of the boundary conditions using expressions like Eq. (66); the requirement that each $Y_{l m}$ component (for $l \leq l_{\max }$ ) of this residual vanishes at the radial collocation points results in a system of algebraic equations for the coefficients $\hat{\psi}_{l m n}$. For the problem considered here these algebraic equations are linear, and with suitable preconditioning are solved using standard numerical methods like GMRES. The elliptic solver is described in detail in Ref. [38].

We use no filtering on the radial basis functions, but apply a rather complicated filtering rule for the angular functions. When evaluating the right side of Eq. (68), we set to zero the coefficients of the terms with $\ell=\ell_{\max }$ in the expansions of the scalars, $\partial_{t} \psi$ and $\partial_{t} \Pi$. The vector $\partial_{t} \Phi_{i}$ is filtered by transforming its components to a vector spherical harmonic basis, setting to zero the coefficients of the terms with $\ell=\ell_{\max }$ in this basis, and then transforming back to Cartesian components. The result $\psi$ of each elliptic solve and the projected $\Pi$ [cf. Equation (53)] are filtered similarly. The projected $\Phi_{i}$ is computed via Eq. (54) from the filtered $\psi$. We find no angular instability, such as the one reported in Ref. [8], when we use this filtering method. And we find no significant change in our results for this problem by increasing the value of $\ell_{\max }$ beyond the value $\ell_{\max }=5$.

\section{A. Testing boundary conditions}

In this section we describe the results of three numerical simulations that explore the effects of boundary conditions on the evolution of the constraints in the scalar field system. First we evolve the initial data in Eqs. (60)(62) using the standard representation of the scalar field system $\left(\gamma_{1}=\gamma_{2}=0\right)$, and using the standard freezing boundary conditions on the incoming fields. We use no constraint projection in this initial simulation. At the inner boundary of the computational domain, $r=r_{\min }=$ $1.9 \mathrm{M}$, all of the fields are outgoing and so no boundary condition is needed there on any of the fields. At the outer boundary, $r=r_{\max }=11.9 M$, the fields $Z^{1}, Z_{i}^{2}$ and $U^{1-}$ are all incoming since the shift points out of the computational domain there: $n_{k} N^{k}=2 M / r$. So we impose the freezing boundary conditions $0=\partial_{t} Z^{1}=\partial_{t} Z_{i}^{2}=\partial_{t} U^{1-}$ on these fields. The results of this first numerical simulation are depicted in Figs. 1 and 2.

Figure 1 illustrates the evolution of the constraints, which we measure using the quantity $\|C(t)\|$,

$$
\|C(t)\|^{2}=\int\left(C_{i} C^{i}+C_{i j} C^{i j}\right) g^{1 / 2} d^{3} x,
$$

divided by a suitable normalization. The constraints in this system are combinations of the derivatives of the dynamical fields. So we normalize the curves in this figure by the quantity $\|\nabla u(t)\|$, which is the natural coordinate-invariant $L^{2}$ measure of the derivatives of the dynamical fields:

$$
\|\nabla u(t)\|^{2}=\int g^{i j} \nabla_{i} u^{\alpha} \nabla_{j} u^{\beta} S_{\alpha \beta} g^{1 / 2} d^{3} x .
$$

The ratio of these quantities, $\|C(t)\| /\|\nabla u(t)\|$, is therefore a meaningful dimensionless measure of the magnitude of constraint violations. When the value of this ratio becomes of order unity, the dynamical fields do not sat-

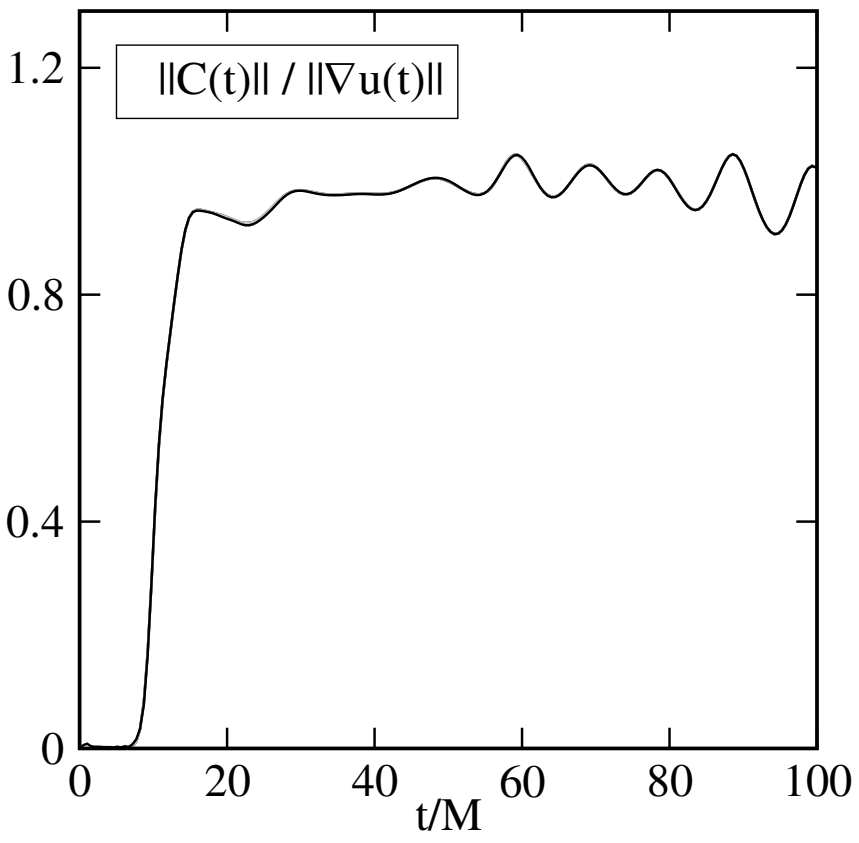

FIG. 1. Constraint violations for evolutions with $\gamma_{1}=\gamma_{2}=$ 0 , freezing boundary conditions, and no constraint projections. Plotted are radial resolutions $N_{r}=21,31, \ldots, 61$; all curves lie on top of each other. 


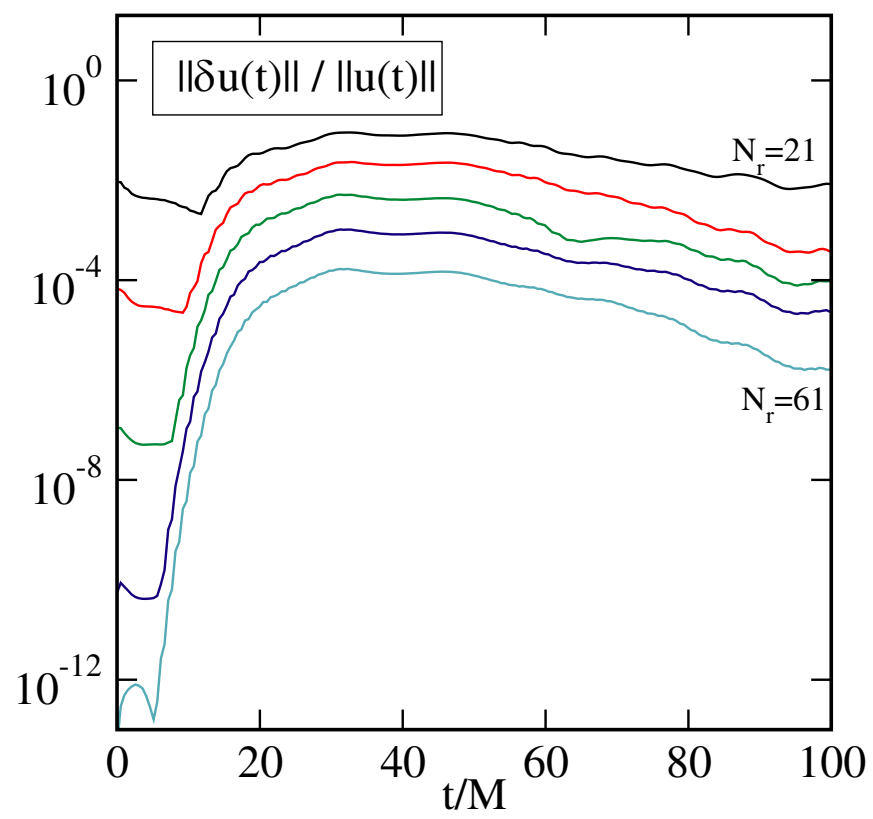

FIG. 2 (color online). Convergence plot for the evolution presented in Fig. 1. Plotted are differences from the solution with radial resolution $N_{r}=81$.

isfy the constraints at all. As we can see in Fig. 1, the constraint-satisfying initial data quickly evolve to a state in which this constraint measure is of order unity. A large increase in constraint violation occurs as the outgoing scalar wave pulse passes through the outer boundary of the computational domain. After this time the numerical solution to the first-order scalar wave system no longer represents a solution to the original scalar field equation.

In Fig. 2 we demonstrate that these numerical solutions are nevertheless numerically convergent. We measure the convergence of these solutions by depicting the quantity

$$
\|\delta u(t)\|^{2}=\int S_{\alpha \beta}\left(u_{N_{r}}^{\alpha}-u_{R}^{\alpha}\right)\left(u_{N_{r}}^{\beta}-u_{R}^{\beta}\right) g^{1 / 2} d^{3} x,
$$

divided by a suitable normalization. This quantity measures the difference between the solution $u_{N_{r}}^{\alpha}$ obtained with radial resolution $N_{r}$, compared to a reference solution $u_{R}^{\alpha}$. In Fig. 2 we use the numerical solution computed with the largest number of radial basis functions $\left(N_{r}=81\right.$ in this case) as the reference solution. In order to make these difference measures meaningful, we normalize them by dividing by an analogous measure of the solution itself:

$$
\|u(t)\|^{2}=\int S_{\alpha \beta} u_{N_{r}}^{\alpha} u_{N_{r}}^{\beta} g^{1 / 2} d^{3} x .
$$

Fig. 2 shows that our computational methods are numerically convergent, even if the solutions are constraintviolating and are therefore unphysical. The rate of convergence of these solutions changes at about $t=10 \mathrm{M}$ because a short wavelength reflected pulse enters the computational domain at about this time. The convergence of these solutions shows that these constraint violations are a feature of the evolution system and the boundary conditions, rather than being artifacts of a poor numerical technique.

Next we evolve the same initial data, Eqs. (60)-(62), using the same standard scalar wave evolution equations $\left(\gamma_{1}=\gamma_{2}=0\right)$, but this time we use constraintpreserving boundary conditions on the fields $Z^{1}$ and $Z_{i}^{2}$, Eqs. (40) and (41). We use no constraint projection in these evolutions. Figure 3 shows that the constraints are in fact satisfied by these solutions to truncation level errors. The solid curves in Fig. 3 show the ratio $\|C(t)\| /\|\nabla u(t)\|$ while the dashed curves show $\|C(t)\| /\|\nabla u(0)\|$. The only difference is that the denominator used for the dashed curves is time independent. The solid curves show that the relative constraint error is approximately constant in time until about $t=40$, at which time a truncation error level constraint-violating pulse from the outer boundary has advected inward across the grid and fallen into the black hole. After $t=40$ the relative constraint error decreases with time. The highestresolution solid curves behave differently: they increase exponentially with time. However, this growth occurs only because the normalization factor in the denominator (which measures the size of the derivatives of the fields) goes to zero as the scalar wave pulse leaves the computa-

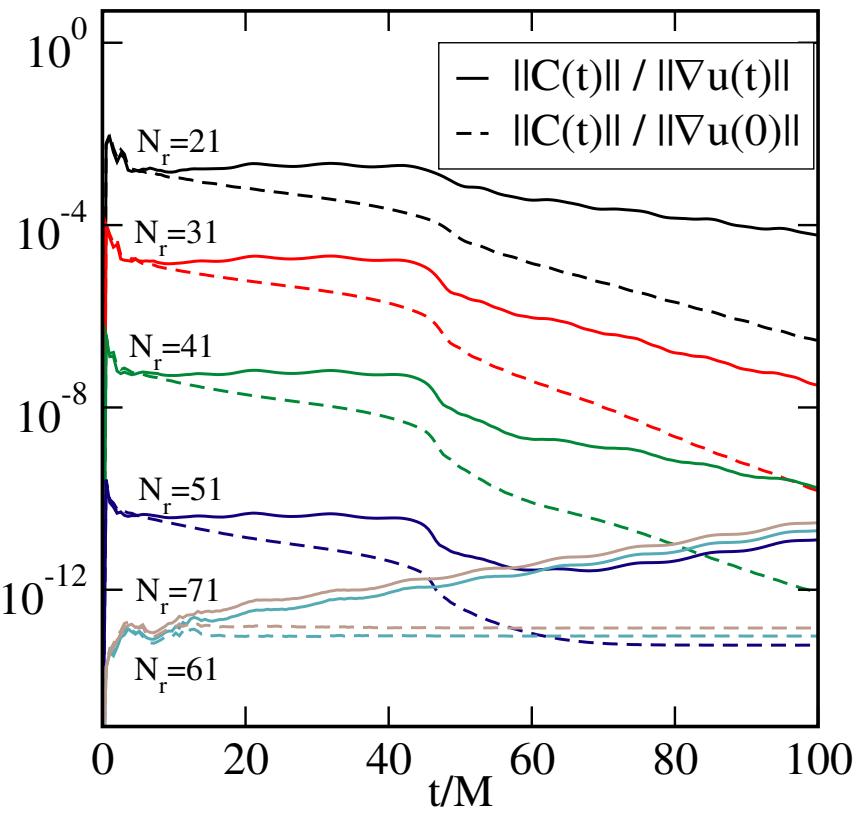

FIG. 3 (color online). Constraint violations for evolutions with $\gamma_{1}=\gamma_{2}=0$, constraint-preserving boundary conditions, and no constraint projection. Solid curves are normalized by the quantity $\|\nabla u(t)\|$ while the dashed curves are normalized by $\|\nabla u(0)\|$. Decay of the normalization factor $\|\nabla u(t)\|$ rather than growth of the constraints causes the growth in the highestresolution solid curves, which have constant roundoff-level constraint violations. 
tional domain. The highest-resolution dashed curves show that the absolute constraint error for these resolutions is constant at roundoff level.

Figure 4 illustrates the numerical convergence of these evolutions. Plotted are the ratios of the differences $\|\delta u(t)\|$ to a measure of the size of the fields. The solid curves in Fig. 4 show the ratio $\|\delta u(t)\| /\|u(t)\|$ while the dashed curves show $\|\delta u(t)\| /\|u(0)\|$. Again, the only difference is that the denominator used for the dashed curves is time independent. Figs. 3 and 4 show that these scalar field evolutions are stable, constraint-preserving and numerically convergent. These solutions therefore represent what we expect to be the correct physical solution to this problem. Were this our only objective, this paper would end here. However our primary interest here is to study the use of projection methods to control the growth of constraints. So we will use the solution found here as a reference to which our later evolutions using constraint projection can be compared.

Our last simulation to study the effects of boundary conditions on the growth of the constraints uses a nonstandard scalar field evolution system with $\gamma_{1}=0$ and $\gamma_{2}=-1 / M$. In other respects, however, this simulation is identical to the one depicted in Figs. 3 and $4:$ It uses the same initial data, Eqs. (60)-(62), the same constraintpreserving boundary conditions, and no constraint projection. Because we use Eq. (39) as a boundary condition

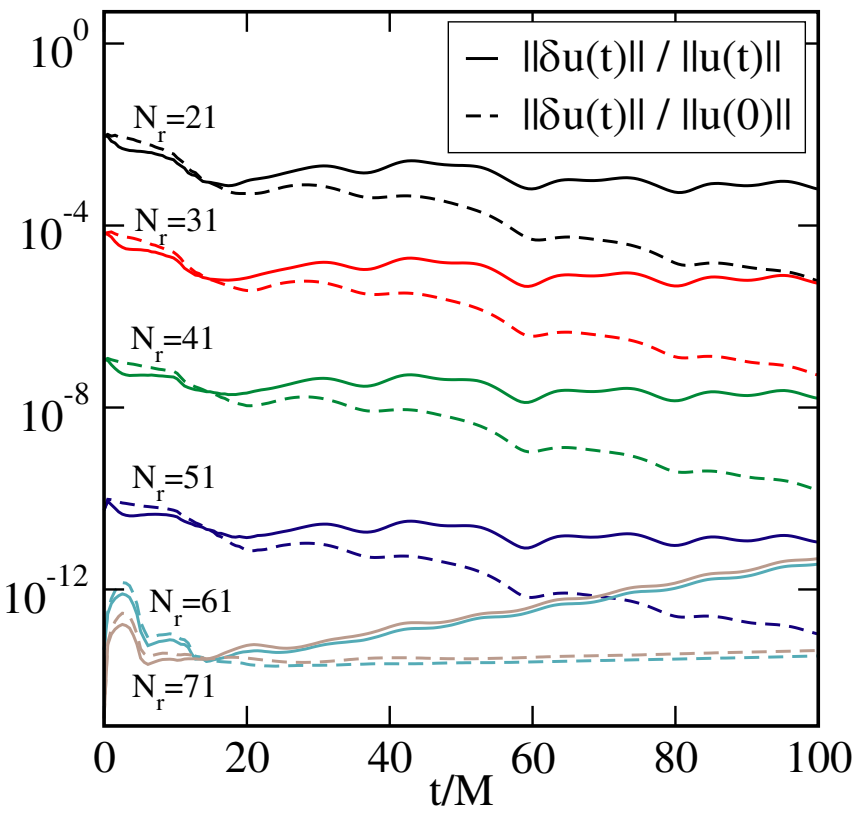

FIG. 4 (color online). Convergence of evolutions shown in Fig. 3. Plotted are differences from the evolution with $N=81$, which is henceforth the reference solution $u_{R}$. Solid curves are normalized by $\|u(t)\|$ while the dashed curves are normalized by $\|u(0)\|$. Decay of the normalization factor $\|u(t)\|$ causes the growth in the highest-resolution solid curves, for which $\|\delta u(t)\|$ is constant at roundoff level. on $U^{1-}$, the constraint-preserving solutions of the equations are the same as those obtained with $\gamma_{1}=\gamma_{2}=0$. However, using an evolution system with $\gamma_{2}=-1 / M$ introduces unstable bulk terms into the constraint evolution equations, Eqs. (29) and (30), so the constraintviolating solutions of the equations will be different. Consequently this system is much more pathological than the standard scalar field system, and provides a much more difficult challenge for the constraint control methods studied here. Figure 5 shows the evolution of the constraints in this system. Truncation level constraint violations in the initial data grow exponentially with an $e$-folding time of approximately $1.1 M$ in these evolutions. The ratio $\|C(t)\| /\|\nabla u(t)\|$ approaches a constant of order unity at late times once the constraint-violating portion of the solution dominates and the denominator begins to grow exponentially as well. The small inset graph in Fig. 5 illustrates that the divergence of these solutions from the reference solution of Fig. 4 grows at the same rate for all spatial resolutions. This suggests that the growth is caused by a constraint-violating solution to the evolution equations rather than a numerical instability.

These evolutions with $\gamma_{2}=-1 / M$ demonstrate that constraint-preserving boundary conditions alone are insufficient to control the growth of constraints in this system. Since the Einstein evolution system is also believed to contain bulk generated constraint violations [2],

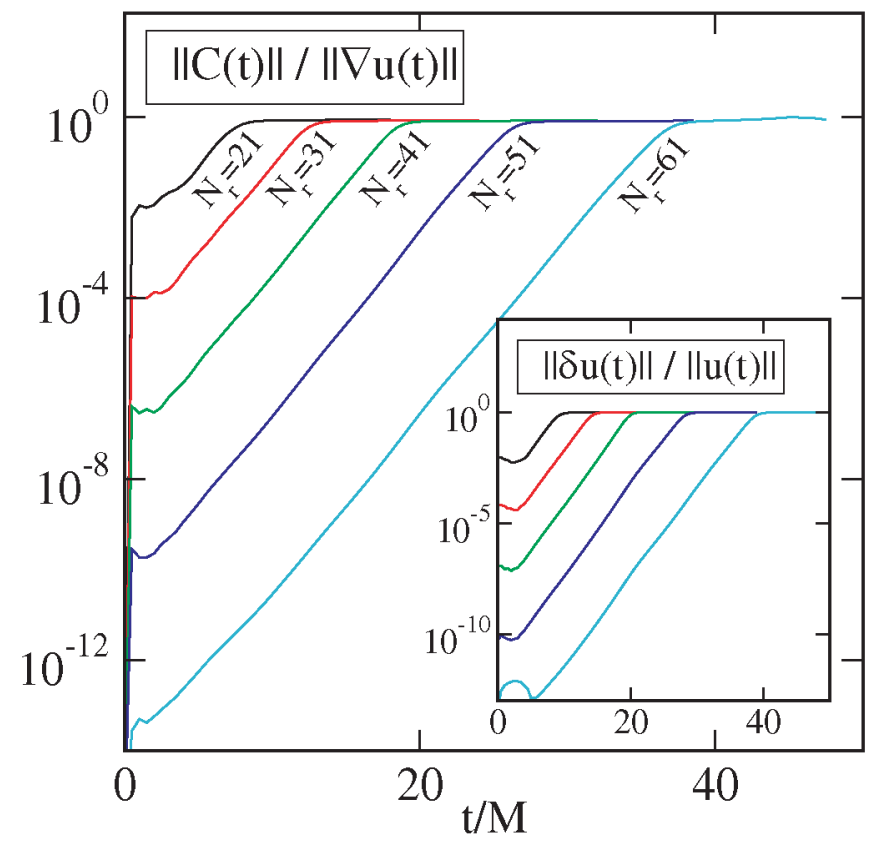

FIG. 5 (color online). Constraint violations for evolutions with $\gamma_{2}=-1 / M$, constraint-preserving boundary conditions, and without constraint projection. The inset shows differences $\|\delta u(t)\| /\|u(t)\|$ from the reference solution of Fig. 4. The curves level off at late times because both numerator and denominator grow exponentially at the same rates. 
this example suggests that constraint-preserving boundary conditions alone will not be sufficient to control the growth of the constraints in the Einstein system.

\section{B. Testing constraint projection}

In this section we discuss two numerical evolutions that explore the use of the constraint projection methods developed in Secs. II and IIIC. The first evolution uses the standard scalar wave evolution system with $\gamma_{1}=$ $\gamma_{2}=0$, and freezing boundary conditions. We have already seen in Figs. 1 and 2 that such evolutions exhibit significant constraint violations once the scalar wave pulse passes through the outer boundary of the computational domain. In this numerical experiment we freely evolve the scalar field to the time $t=20 M$, and then perform a single constraint projection on the solution using Eqs. (52)-(54) with $\Lambda=2 / M$. We then evolve the system freely again to $t=40 \mathrm{M}$. Figure 6 shows how the constraints respond to a single constraint projection. We use a very fine time-scale in Fig. 6, showing in detail the times around $t=20 M$ when the constraint projection is performed. Individual points in Fig. 6 show the amount of constraint violation after each individual time step. The value of the constraints drops sharply at the time step where the constraint projection is performed, and as we expect, the value of the constraints after this projection step is smaller for higher resolutions. So the constraint projection step is successful in significantly reducing the

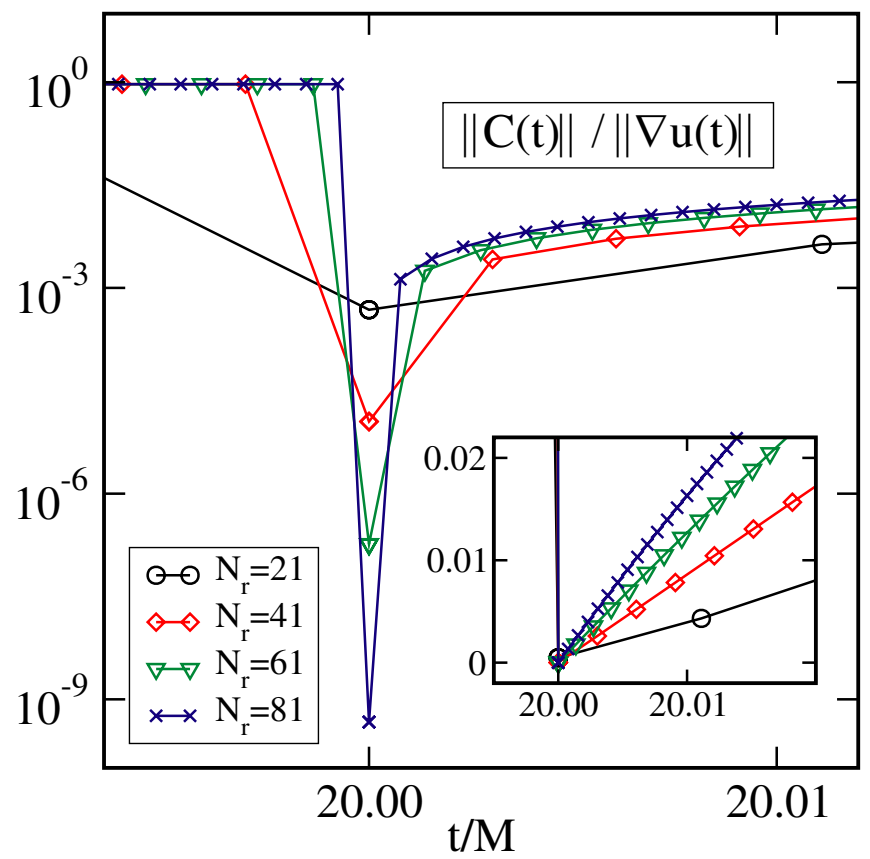

FIG. 6 (color online). Constraint violations for evolutions with $\gamma_{1}=\gamma_{2}=0$, freezing boundary conditions, and a single constraint projection at $t=20 M$ (with $\Lambda=2 / M$ ). Points show $\|C(t)\| /\|\nabla u(t)\|$ after each time step. The inset plots the same data on a linear scale. size of the constraints. But something rather unexpected happens next: the constraints increase by orders of magnitude on the very next free evolution time step after the constraint projection. The small inset in Fig. 6 shows the same data plotted on a linear rather than a logarithmic scale. This shows that the constraints grow linearly in time after the constraint projection step on a very short time-scale.

Figure 7 provides some information about the reason for this strange behavior by showing the convergence of these numerical solutions. For times before the constraint projection step at $t=20 M$, the solutions show good numerical convergence as the number of radial collocation points is increased. But there is a sharp breakdown of numerical convergence (or at least a sharp drop in the rate of numerical convergence) after the constraint projection step.

Figure 8 provides some deeper insight into the reason for this lack of convergence. Plotted in Fig. 8 are a sequence of curves showing the radial dependences of the dipole part of the scalar field $\langle\psi\rangle_{10}$ and the monopole part of the constraints $\left\langle C_{i} C^{i}\right\rangle_{00}$ at a sequence of times including the constraint projection step. The spherical harmonic components of a function $Q$ are defined by

$$
\langle Q\rangle_{l m}=\int Y_{l m}^{*}(\theta, \varphi) Q(r, \theta, \varphi) \sin \theta d \theta d \varphi
$$

The dashed lines at the bottom of Fig. 8 shows the radial profiles at $t=20 \mathrm{M}$ immediately before the constraint projection, while the lowest solid lines show these profiles at the same time $t=20 M$ just after the projection. We see

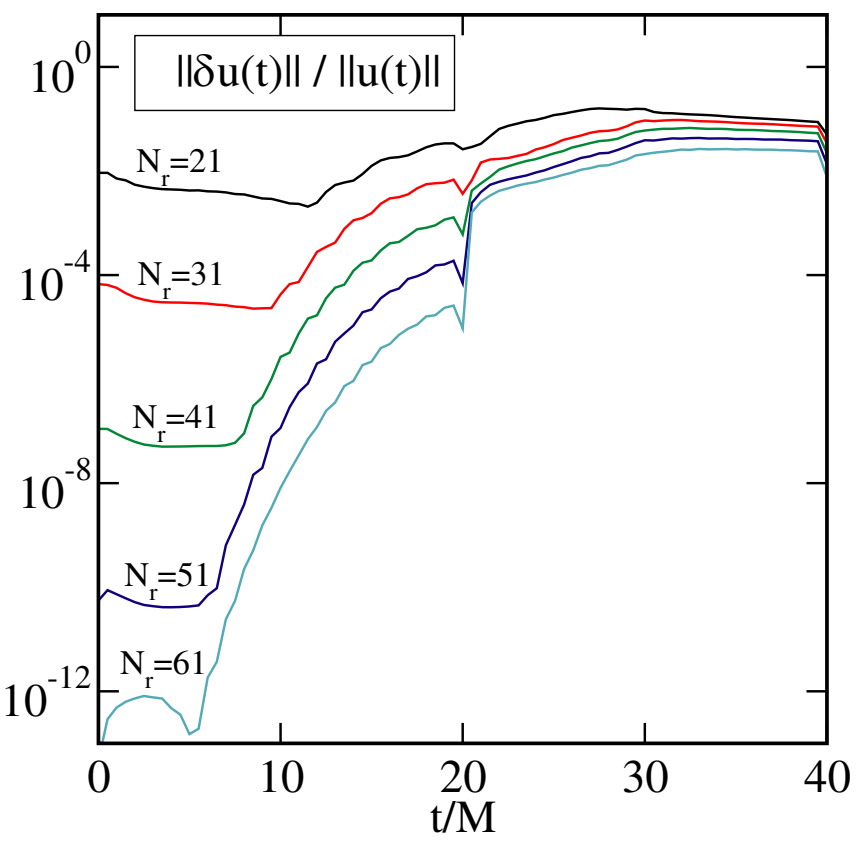

FIG. 7 (color online). Convergence of evolutions shown in Fig. 6. Plotted are differences from the evolution with $N_{r}=81$. 


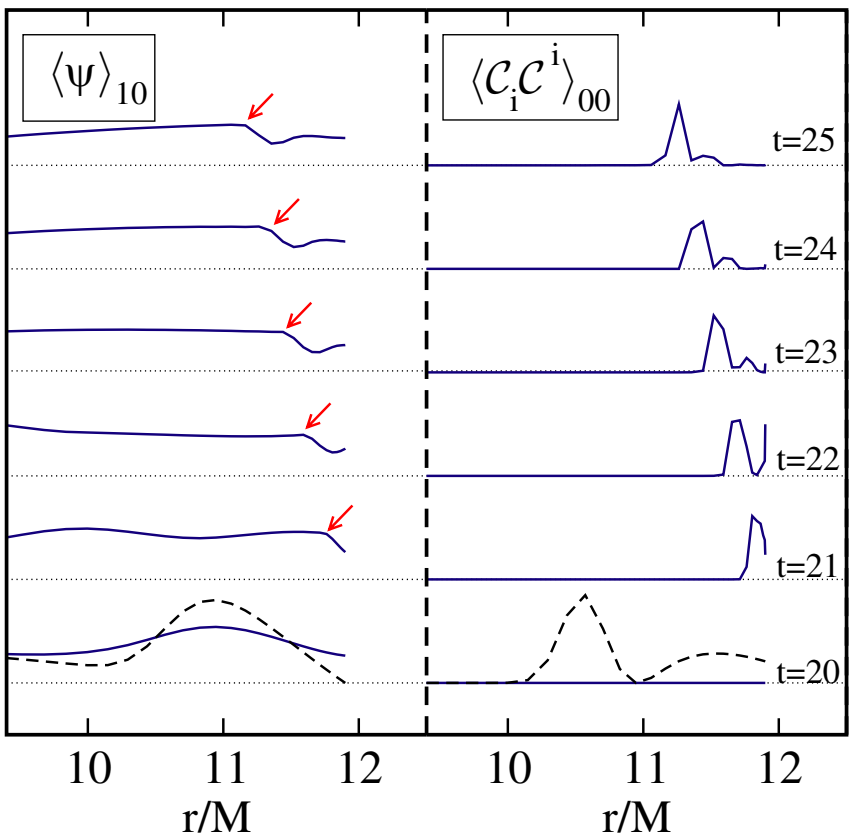

FIG. 8 (color online). Radial profiles of $\langle\psi\rangle_{10}$ and $\left\langle C_{i} C^{i}\right\rangle_{00}$ for the evolution of Fig. 6 . The solid lines represent times $t / M=$ $20, \ldots, 25$. The dashed line represents the state just before the constraint projection at $t / M=20$. The arrows indicate the location of the nonsmoothness in $\psi$.

that the constraints essentially vanish after the constraint projection step. The next profile at $t=21 \mathrm{M}$ shows that the scalar field develops some nonsmooth radial structure immediately after the projection step, which subsequently propagates into the computational domain. This nonsmoothness in $\psi$ causes a sharp spike in the constraints, seen clearly in Fig. 8. Spectral methods do not converge well for nonsmooth functions, so the emergence of this structure in $\psi$ explains the breakdown in the numerical convergence and then the breakdown in our constraint projection method. The emergence of the nonsmoothness in $\psi$ seems to be caused by the constraint projection step in the following way: The projection produces a $\psi$ that is nonvanishing at the boundary, and the freezing boundary condition then forces $\psi=Z^{1}$ (and $Z_{i}^{2}$ ) to develop kinks (see Ref. [8]) which propagate into the computational domain during the free evolution steps following the projection.

Figs. 6-8 demonstrate that constraint projection is not successful in removing large constraint violations when used in conjunction with freezing boundary conditions. One might hope that this failure could be corrected by projecting out the constraints before they are allowed to grow too large. Figure 9 shows the convergence of solutions in which a constraint projection is performed after each evolution time step, for a variety of different time steps $\Delta t$. Like the evolutions shown in Figs. 6-8, these evolutions use the standard scalar field system $\left(\gamma_{1}=\right.$ $\gamma_{2}=0$ ), freezing boundary conditions, and constraint

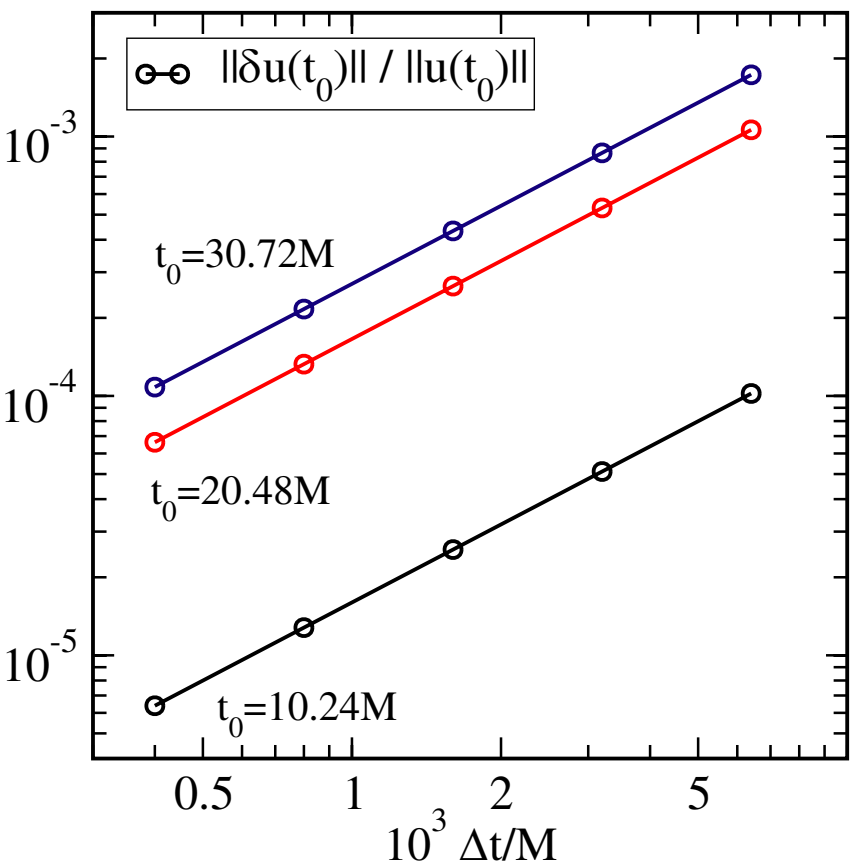

FIG. 9 (color online). Differences between evolutions with time step $\Delta t$ and the reference solution $u_{R}$ (of Fig. 4) at fixed evolution times $t_{0}$. Evolutions use $\gamma_{1}=\gamma_{2}=0$, freezing boundary conditions, and constraint projection with $\Lambda=$ $2 / M$ after each time step, $\Delta T=\Delta t$.

projection with $\Lambda=2 / M$. The three curves in Fig. 9 measure the convergence of the solution (relative to the highest-resolution reference solution depicted in Fig. 4) at three different times in this evolution, $t_{0}=10.24 M$, $20.48 M$, and $30.72 M$. All of these evolutions use the same spatial resolution, $N_{r}=51$. These graphs show that the convergence towards the reference solution is only first-order in the time step $\Delta t$. This convergence is significantly worse than that expected for the fourthorder Runge-Kutta time step integrator that we use. In contrast the free evolutions with constraint-preserving boundary conditions shown in Fig. 4 achieve $\left\|\delta u\left(t_{0}\right)\right\| /$ $\left\|u\left(t_{0}\right)\right\| \lesssim 10^{-10}$ with a timestep similar to the largest $\Delta t$ shown in Fig. 9. We conclude that constraint projection produces only first-order in time convergent numerical solutions when used in conjunction with standard freezing boundary conditions, and is therefore an ineffective substitute for constraint-preserving boundary conditions.

Finally we apply constraint projection to the pathological scalar wave evolution system $\left(\gamma_{1}=0\right.$ and $\gamma_{2}=$ $-1 / M)$, which we failed to control with constraintpreserving boundary conditions alone. We project every $\Delta T=2 M$ using $\Lambda=\sqrt{2} / M$, and we continue to use constraint-preserving boundary conditions. Except for constraint projection, this is the same as the evolution shown in Fig. 5. Figure 10 shows that the constraints are reduced to truncation error levels in these evolutions. The small inset graph shows these same curves with a finer 


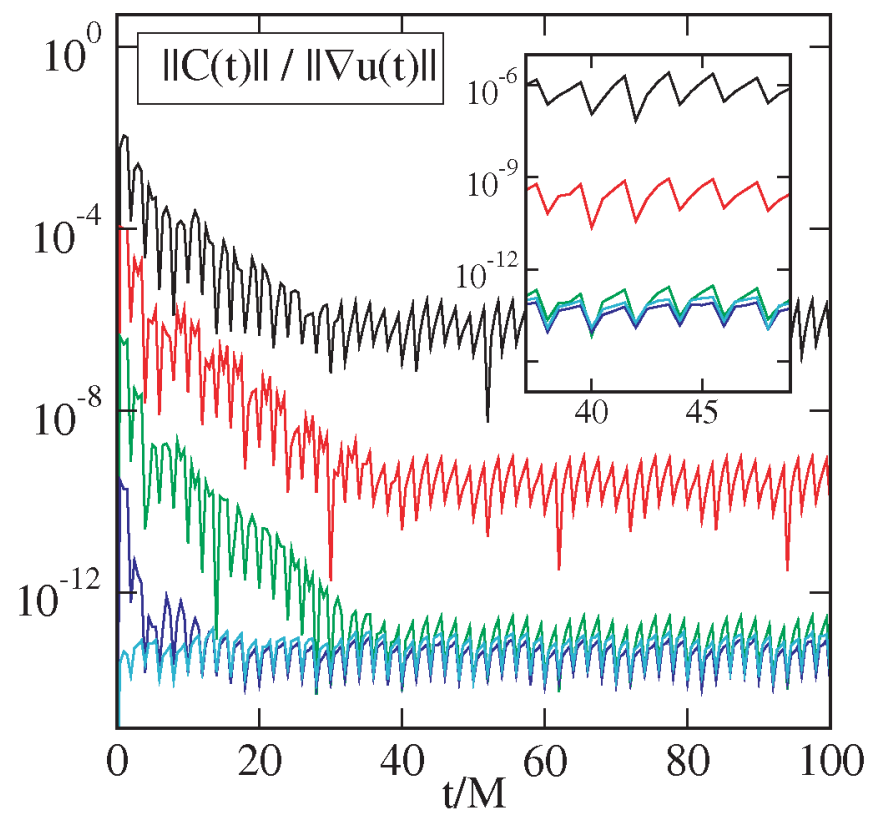

FIG. 10 (color online). Constraint violations $\|C(t)\| /$ $\|\nabla u(t)\|$ for evolutions with $\gamma_{1}=0$ and $\gamma_{2}=-1 / M$, constraint-preserving boundary conditions, and constraint projection with $\Lambda=\sqrt{2} / M$ every $\Delta T=2 M$. Inset shows the same data with finer time resolution.

time resolution, so the sawtooth shaped evolution of the constraints can be seen more clearly. We note that constraint projection does not occur at every evolution time step in these simulations, but rather at fixed times separated by $\Delta T=2 M$. The evolutions with the finest spatial resolution take more than 1000 time steps between pro-

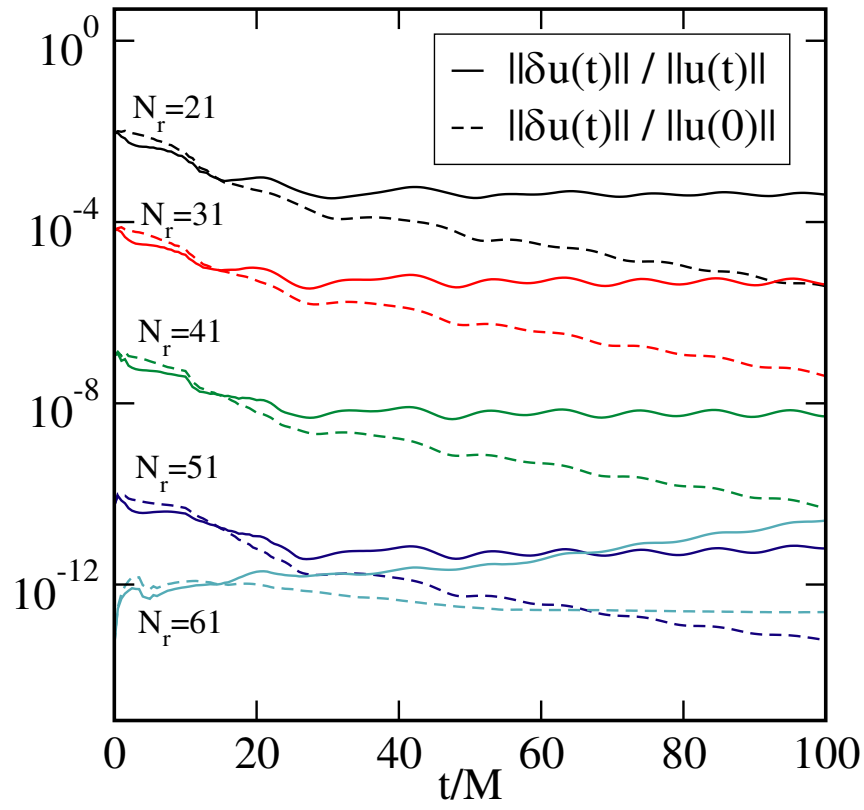

FIG. 11 (color online). Differences from the reference solution $u_{R}$ (of Fig. 4) for the evolutions shown in Fig. 10. jections. Figure 11 shows the convergence between these evolutions and the highest-resolution reference solution depicted in Fig. 4. This figure demonstrates that the constraint projection method combined with constraintpreserving boundary conditions succeeds in producing the same numerical solution as our reference solutioneven for this pathological scalar field system.

\section{Optimizing constraint projection}

In this section we explore ways to optimize the use of the constraint projection methods developed in Secs. II and III C. In particular we investigate how important the choice of the parameter $\Lambda$ is to the effectiveness of the projection, and we determine its optimal value. We also vary the time between projection steps, $\Delta T$, and determine the optimal rate at which to perform these projections. Finally we measure the computational cost of performing a scalar field evolution with constraint projection, compared to the cost of doing a free evolution.

Figure 12 shows convergence plots for evolutions of the pathological scalar field system with $\gamma_{1}=0$ and $\gamma_{2}=$ $-1 / M$, constraint-preserving boundary conditions, and constraint projection every $\Delta T=2 M$. All evolutions use the same radial resolution, $N_{r}=41$. Each of the solid curves in Fig. 12 represents an evolution using a different choice of the parameter $\Lambda$. We see that the evolutions using projections with $\Lambda=\sqrt{2} / M$ are somewhat closer to the reference solution than the others, but the size of the differences are not very sensitive to the value of $\Lambda$. The

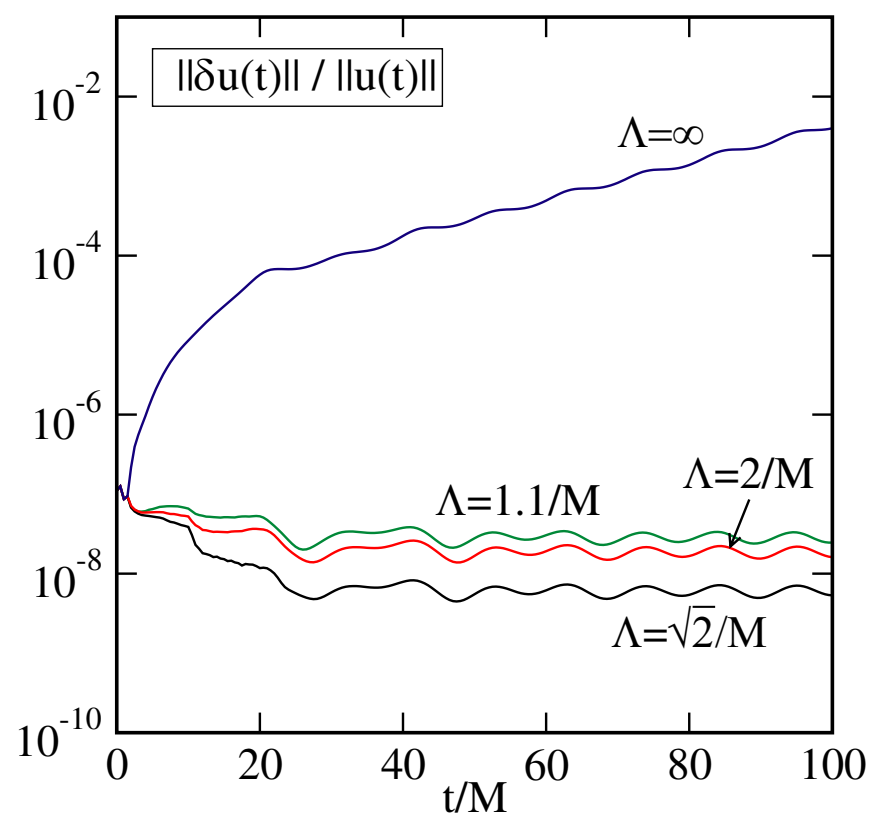

FIG. 12 (color online). Differences $\|\delta u(t)\| /\|u(t)\|$ from the reference solution $u_{R}$ of Fig. 4 are plotted for different choices of $\Lambda$. Evolutions with $\gamma_{1}=0$ and $\gamma_{2}=-1 / M$, constraintpreserving boundary conditions, constraint projection every $\Delta T=2 M$ 
only projected solution having significantly worse accuracy than the others is the one with $\Lambda=\infty$, which corresponds to the simple projection with $\psi=\bar{\psi}, \Pi=\bar{\Pi}$ and $\Phi_{i}=\partial_{i} \psi$. For all choices of $\Lambda$, including $\Lambda=\infty$, these evolutions are exponentially convergent with increasing $N_{r}$.

We have some understanding of why there is an optimal choice for the parameter $\Lambda$ : It is possible to analyze the projection process completely and analytically for scalar field evolutions with a flat background metric on a computational domain with three-torus $\left(T^{3}\right)$ topology. By performing a Fourier transform of the fields in this case it is easy to show that the fields break up into modes that propagate with the usual dispersion relation $\omega^{2}=\vec{k} \cdot \vec{k}$, plus others that grow exponentially in time with dispersion relation $\omega=i \gamma_{2}$. The projection step becomes a simple algebraic transformation on the Fourier components of the field in this case. So it is straightforward to show that the projection step completely removes the modes that grow exponentially with time only when the parameters satisfy $\Lambda^{2}=2 \gamma_{2}^{2}$. For evolutions on computational domains with different topologies, and different background metrics, it is not possible to determine the optimal choice of $\Lambda$ using such a simple argument. However it is not surprising that the optimal choice is not too different from $\Lambda^{2}=2 \gamma_{2}^{2}$.

Next we consider the effect of varying the times between constraint projections. Figure 13 shows the con-

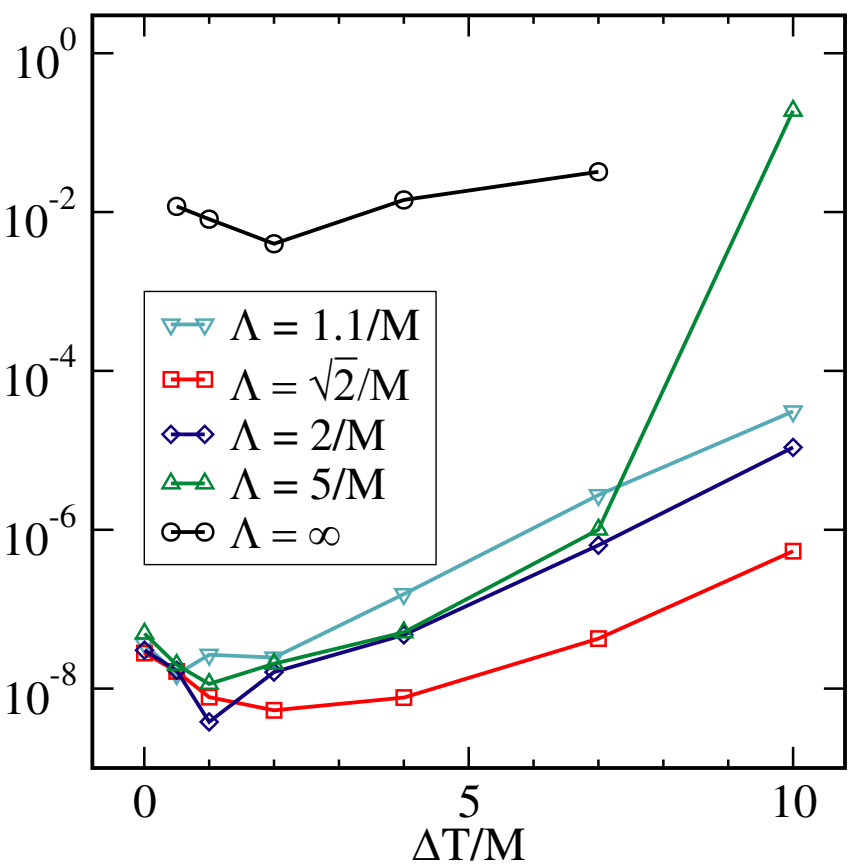

FIG. 13 (color online). Evolutions with $\gamma_{1}=0$ and $\gamma_{2}=$ $-1 / M$, constraint-preserving boundary conditions and constraint projection every $\Delta T$. Differences from the reference solution $u_{R}$ (of Fig. 4) at $t_{0}=100 M$ for different choices of $\Delta T$ and $\Lambda$. vergence measure $\left\|\delta u\left(t_{0}\right)\right\| /\left\|u\left(t_{0}\right)\right\|$ for evolutions of the pathological scalar field system with $\gamma_{1}=0$ and $\gamma_{2}=$ $-1 / M$, constraint-preserving boundary conditions, and constraint projections with various values of $\Lambda$ and $\Delta T$. These evolutions are all carried out with the same radial resolution $N_{r}=41$, and are compared with the reference solution of Fig. 4 at the time $t_{0}=100 M$. Each curve in Fig. 13 represents a set of evolutions with the same value of $\Lambda$ but varying $\Delta T$. The smallest $\Delta T$ for each curve corresponds to projecting at each evolution time step. We see that all of these curves show a minimum difference with the reference solution, and this minimum occurs at about $\Delta T \approx 1 M$ in all of these curves. This coincides with the $e$-folding time of the bulk constraint violations, $-1 / \gamma_{2}$; hence we expect that constraint projection should generally be applied on a time-scale comparable to that of the constraint growth. Figure 13 also reveals that projections performed with $\Lambda^{2}=2 \gamma_{2}^{2}$ are the optimal ones over a fairly broad range of projection times $\Delta T$. The evolutions with simple constraint projection $(\Lambda=\infty)$ crash for very small values of $\Delta T$, as well as for $\Delta T=10 M$.

Finally, we have made some measurements to evaluate the computational cost of doing scalar field evolutions with constraint projection, compared to the cost of free evolution. Figure 14 contains two curves that measure the computational cost of doing optimal projection with $\Delta T=2 M$. The solid curve shows the ratio of the time the code spends doing the constraint projection step (i.e. doing the elliptic solve) $T_{\text {ell }}$ with the time the code spends doing evolution steps $T_{\text {hyp }}$. This ratio decreases from about 0.1 using a very coarse spatial resolution to about

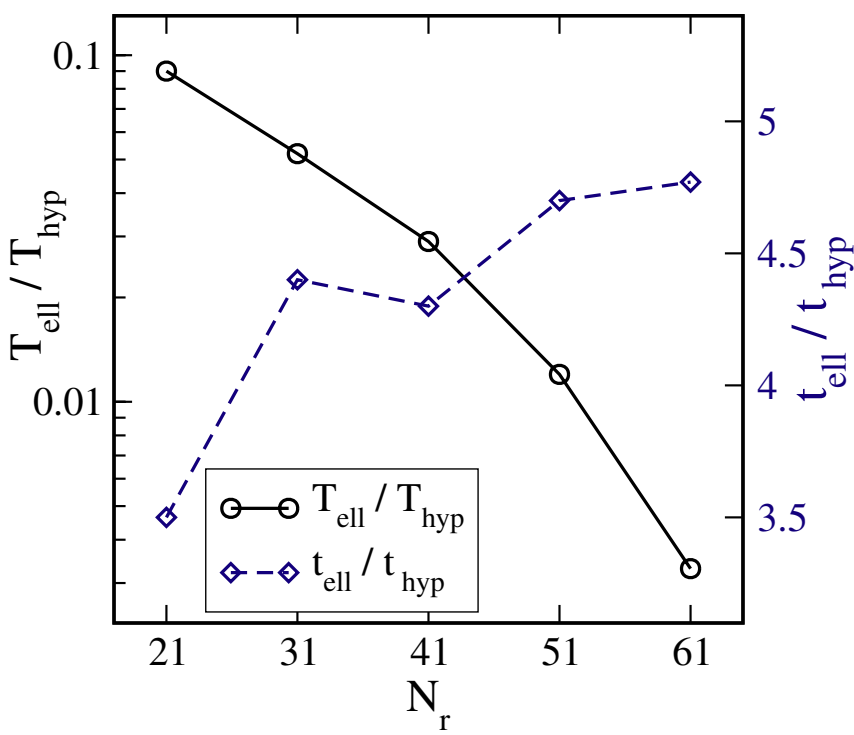

FIG. 14 (color online). Solid curve (left axis) shows the ratio of time spent in elliptic solves to time spent in the hyperbolic evolution code. Dashed curve (right axis) shows the ratio of time required for one elliptic solve to the time for one evolution time step. 
0.003 using a very fine spatial resolution. The ratio $T_{\text {ell }} / T_{\text {hyp }}$ decreases when the spatial resolution is increased because the code must take many more free evolution time steps in the time $\Delta T$ between projection steps in this case. The dashed curve in Fig. 14 measures the ratio of the time needed to perform one constraint projection, $t_{\text {ell }}$, with the time needed to take one free evolution step, $t_{\text {hyp }}$. We see that this ratio is fairly independent of resolution using our spectral elliptic solver, and ranges from about 3.5 at low spatial resolution to about 5 at high resolution. These tests show that the computational cost of performing constraint projection is only a small fraction of the total computational cost of performing these scalar field evolutions. We conclude that computational cost should not be used as an argument against the use of constraint projection methods.

\section{DISCUSSION}

We have developed general methods in Sec. II for constructing optimal projection operators that map the dynamical fields of hyperbolic evolution systems onto the constraint submanifold associated with these systems. These methods are worked out explicitly in Sec. III for the case of a new evolution system that describes the propagation of a scalar field on a fixed background spacetime. The constraint projection map for this system requires the solution of one elliptic partial differential equation each time a projection is performed. The new scalar field system introduced in Sec. III has the interesting property that it suffers from constraint violations that flow into the domain through timelike boundaries and also from violations generated by bulk terms in the equa- tions. So this system exhibits both types of constraintviolating pathologies that can occur in the Einstein evolution system. To test our constraint projection methods, we perform a number of numerical evolutions of this scalar field system propagating on a black hole spacetime. We show that constraint-preserving boundary conditions alone are not capable of controlling the growth of constraints in this scalar field system. Constraint projection is also shown to be ineffective when used in conjunction with traditional boundary conditions that do not prevent the influx of constraint violations through the boundary. However we show that the combination of constraint projection and constraint-preserving boundary conditions is a very effective method for controlling the growth of the constraints. We measure the computational cost of performing these constraint projections and show that at the highest numerical resolutions, the projections account for only a fraction of a percent of the total computational cost of the evolution. Thus high computational cost can no longer be cited as a reason to avoid constraint projection techniques.

\section{ACKNOWLEDGMENTS}

We thank Saul Teukolsky and Manuel Tiglio for helpful comments. Some of the computations for this project were performed with the Tungsten cluster at NCSA. This work was supported in part by NSF Grants Nos. PHY-0099568, PHY-0244906 and NASA Grant Nos. NAG5-10707, NAG5-12834 at Caltech, NSF Grant Nos. DMS-9875856, DMS-0208449, DMS-0112413 at UCSD, and NSF Grant Nos. PHY-9900672, PHY0312072 at Cornell.
[1] L. E. Kidder, M. A. Scheel, and S. A. Teukolsky, Phys. Rev. D 64, 064017 (2001).

[2] L. Lindblom and M. A. Scheel, Phys. Rev. D 66, 084014 (2002).

[3] M. A. Scheel, L. E. Kidder, L. Lindblom, H. P. Pfeiffer, and S. A. Teukolsky, Phys. Rev. D 66, 124005 (2002).

[4] M. S. Iriondo and O. A. Reula, Phys. Rev. D 65, 044024 (2002).

[5] B. Szilágyi, B. Schmidt, and J. Winicour, Phys. Rev. D 65, 064015 (2002).

[6] G. Calabrese, L. Lehner, and M. Tiglio, Phys. Rev. D 65, 104031 (2002).

[7] B. Szilágyi and J. Winicour, Phys. Rev. D 68, 041501 (2003).

[8] L. Lindblom, M. A. Scheel, L. E. Kidder, H. P. Pfeiffer, D. Shoemaker, and S. A. Teukolsky, Phys. Rev. D 69, 124025 (2004).

[9] J. M. Stewart, Classical Quantum Gravity 15, 2865 (1998).
[10] H. Friedrich and G. Nagy, Commun. Math. Phys. 201, 619 (1999).

[11] G. Calabrese, J. Pullin, O. Sarbach, M. Tiglio, and O. Reula, Commun. Math. Phys. 240, 377 (2003).

[12] S. Frittelli and R. Gomez, Classical Quantum Gravity 20, 2379 (2003).

[13] S. Frittelli and R. Gomez, Phys. Rev. D 68, 044014 (2003).

[14] G. Calabrese and O. Sarbach, J. Math. Phys. (N.Y.) 44, 3888 (2003).

[15] S. Frittelli and R. Gomez, Phys. Rev. D 69, 124020 (2004).

[16] A. M. Alekseenko, gr-qc/0405080.

[17] R. F. Stark and T. Piran, Phys. Rev. Lett. 55, 891 (1985).

[18] A. M. Abrahams and C. R. Evans, Phys. Rev. D 46, 4117 (1992).

[19] M.W. Choptuik, Phys. Rev. Lett. 70, 9 (1993).

[20] A. M. Abrahams and C. R. Evans, Phys. Rev. Lett. 70, 2980 (1993). 
[21] A. M. Abrahams, G. B. Cook, S. L. Shapiro, and S. A. Teukolsky, Phys. Rev. D 49, 5153 (1994).

[22] M.W. Choptuik, E.W. Hirschmann, S. L. Liebling, and F. Pretorius, Classical Quantum Gravity 20, 1857 (2003).

[23] M.W. Choptuik, E.W. Hirschmann, S. L. Liebling, and F. Pretorius, Phys. Rev. D 68, 044007 (2003).

[24] S. Bonazzola, E. Gourgoulhon, P. Grandclement, and J. Novak, gr-qc/0307082.

[25] M. Anderson and R. A. Matzner, gr-qc/0307055.

[26] E. Schnetter, Ph.D. thesis, Universität Tübingen, 2003, http://w210.ub.uni-tuebingen.de/dbt/volltexte/2003/819/.

[27] E. Zeidler, Nonlinear Functional Analysis and its Applications (Springer-Verlag, New York, 1991), Vol. III.

[28] C. Lanczos, The Variational Principles of Mechanics (Dover Publications, Inc., New York, 1949).

[29] V.I. Arnold, Mathematical Methods of Classical Mechanics (Springer-Verlag, New York, 1989).

[30] J.E. Marsden and T.J. R. Hughes, Mathematical Foundations of Elasticity (Dover Publications, New York, 1994).

[31] C.W. Gear, IEEE Trans. Circuit Theory CT-18, 89 (1971).

[32] B. Leimkuhler and S. Reich, Mathematics of Computation 63, 589 (1994).

[33] B. Leimkuhler and R. D. Skeel, J. Comput. Phys. 112, 117 (1994).
[34] B. Leimkuhler, Symplectic methods for rigid body dynamics, Lecture Notes in Computational Science and Engineering Vol. 4 (Springer-Verlag, Berlin, 1998).

[35] E. Hairer, C. Lubich, and G. Wanner, Geometric Numerical Integration (Springer-Verlag, Berlin, 2001).

[36] R. Temam, Navier-Stokes Equations: Theory and Numerical Analysis (North-Holland, New York, 1977).

[37] F. Brezzi and M. Fortin, Mixed and Hybrid Finite Element Methods (Springer-Verlag, New York, 1991).

[38] H. P. Pfeiffer, L. E. Kidder, M. A. Scheel, and S. A. Teukolsky, Comput. Phys. Commun. 152, 253 (2003).

[39] M. E. Taylor, Partial Differential Equations, (SpringerVerlag, New York, 1996), Vol. III.

[40] B. Gustafsson, H.-O. Kreiss, and J. Oliger, Time Dependent Problems and Difference Methods, (Wiley, New York, 1995).

[41] M. A. Scheel, A. L. Erickcek, L. M. Burko, L. E. Kidder, H. P. Pfeiffer, and S. A. Teukolsky, Phys. Rev. D 69, 104006 (2004).

[42] J. Kevorkian, Partial Differential Equations (Wadsworth \& Brooks/Cole Advanced Books \& Software, Pacific Grove, 1990).

[43] M. E. Taylor, Partial Differential Equations, (SpringerVerlag, New York, 1996), Vol. I.

[44] L. E. Kidder, M. A. Scheel, S. A. Teukolsky, E. D. Carlson, and G. B. Cook, Phys. Rev. D 62, 084032 (2000).

[45] M. Bjørhus, SIAM J. Sci. Comput. 16, 542 (1995). 